\title{
Mental health of people detained within the justice system in Africa: systematic review and meta-analysis
}

\author{
Aish Lovett ${ }^{1}$, Hye Rim Kwon ${ }^{2}$, Khameer Kidia ${ }^{3,4^{*}}$, Debra Machando ${ }^{3,5}$, Megan Crooks ${ }^{6}$, Gregory Fricchione ${ }^{7}$, \\ Graham Thornicroft ${ }^{8}$ and Helen E. Jack J $^{3,8,9}$
}

\begin{abstract}
Worldwide, people with mental disorders are detained within the justice system at higher rates than the general population and often suffer human rights abuses. This review sought to understand the state of knowledge on the mental health of people detained in the justice system in Africa, including epidemiology, conditions of detention, and interventions. We included all primary research studies examining mental disorders or mental health policy related to detention within the justice system in Africa. 80 met inclusion criteria. $67 \%$ were prevalence studies and metaanalysis of these studies revealed pooled prevalence as follows: substance use 38\% (95\% Cl 26-50\%), mood disorders $22 \%$ (95\% Cl 16-28\%), and psychotic disorders 33\% (95\% Cl 28-37\%). There were only three studies of interventions. Studies examined prisons (46\%), forensic hospital settings (37\%), youth institutions (13\%), or the health system (4\%). In $36 \%$ of studies, the majority of participants had not been convicted of a crime. Given the high heterogeneity in subpopulations identified in this review, future research should examine context and population-specific interventions for people with mental disorders.
\end{abstract}

\section{Introduction}

Investments in mental health care and research are critical: globally, psychiatric and neurological disorders comprise approximately $13 \%$ of the disability-adjusted life years and nearly one-third of years lived with disability [1]. The prevalence of mental illness is higher among people detained within the justice system (PDJS) compared to the general population [2-9]. In this review, PDJS are defined as people detained or incarcerated in prisons, jails, youth institutions, or forensic inpatient units of hospitals. In accordance with the World Health Organization (WHO)'s definition, a forensic inpatient unit is "exclusively maintained for the evaluation or treatment of people with mental disorders who are involved with the justice system. These units can be located in mental hospitals, general hospitals, or elsewhere." [10]. This review uses an inclusive definition of detention to respond to

\footnotetext{
*Correspondence: kkidia@gmail.com

${ }^{3}$ Kushinga, 8 Collina Close, Borrowdale, Harare, Zimbabwe

Full list of author information is available at the end of the article
}

the scarcity of research on PDJS's mental health in Africa, particularly for people detained outside of prisons. International systematic reviews on the mental health of PDJS show that populations in prisons are multiple times more likely to have several major mental disorders $[2,4,5,9]$ and have a three to sixfold higher risk of death by suicide [3]. More than 10 million people are held in penal institutions worldwide [11], and have increased risk of adverse outcomes such as all-cause mortality, suicide, self-harm, violence, and victimization [3]. The prevalence of mental disorders in African countries is of particular concern due to resource constraints for mental health and expansion of the movement to shift resources from institutions to community-based care in low- and middle-income countries (LMICs) [12, 13]. In 2017, there were 2.5 total mental health beds per 100,000 population in African countries as a whole, $80 \%$ of which were in psychiatric hospitals [10], illustrating a contrast between where most existing mental health resources go (hospitals) and where services may be needed and, in reality, delivered (the community). There is, however, an international movement, including 
in Africa, to shift resources from a country's psychiatric hospital or hospitals to other forms of mental health services [14, 15]. This movement recognizes that most people in low-resource settings have been receiving care in the community (if at all). However, in contexts with inadequate community health care, people previously in institutions may face increased risk of diversion to the justice system [15-18]. As health resources shift to better support mental health care in primary care and outpatient services in some African countries, research is needed on mental health interventions for justice-involved populations with elevated prevalence rates.

However, there has been minimal attention to the mental health of PDJS in international data collection and guidelines [19]. We surveyed United Nations (UN) and WHO guidelines on mental health and detained populations from the past 15 years (Table 1) and found that the mental health of PDJS has not been present in the majority of publications. The exclusion of the justice system from research or policy priorities contrasts to consensus in international prison literature which explicitly requires extensive mental health care services (see Table 1).

Despite the burden of mental disorders among PDJS in Africa, research in this area is sparse [3, 19]. Existing international systematic reviews on the mental health of PDJS have either included studies in only one African country, Nigeria [2], or none at all [4, 5, 20,21]. Moreover, these reviews do not report methodological bias or ethics procedures data of included studies, and the search criteria of most do not include institutions that detain justiceinvolved youth or inpatients in forensic psychiatry units $[2,4,5,21]$, even though this is where many people with mental illness may be detained and are similar to prisons in some countries [22]. A recent systematic review on the influence of prison climate on mental health resulted in studies from only high-income countries [21]. Similarly, a review of psychological therapies for PDJS internationally did not result in studies from LMIC countries other than India, Iran, and China [20]. Importantly, however, its search criteria included youth and participants in secure hospitals, while the other systematic reviews include only those in prisons and jails [2, 4, 5, 21].

Given the paucity of research focused on Africa and the resource constraints of these countries, this review aims to understand the scope of knowledge on the mental health of PDJS in Africa and to identify gaps in the literature, in order to inform future research, interventions, harm reduction, efforts, and policy. Given our study aims, we did not limit our search and selection to a single study design or outcome measure. In contrast to previous reviews investigating only prevalence, specific study types, or restricted to prisons, the broad scope of this review responds to the scarcity of research in African contexts and the systems-wide nature of detention and issues surrounding the mental health of PDJS.

\section{Methods}

The review followed guidelines of the Preferred Reporting Items for Systematic Review and Meta-analyses (PRISMA) [23], and the meta-analysis of observational studies in epidemiology (MOOSE) [24], both of which are found in Additional file 1: Appendices S1 and S2. The protocol was registered in the International Prospective Register of Systematic Reviews (PROSPERO). The Registration Number is CRD42018098852.

\section{Search strategy and selection criteria}

We searched PubMed, PsycINFO, Embase, Web of Science, and Africa Index Medicus for studies dating from the inception of the database to 16 November 2017, and published in English or French, major research languages on the African continent. Because the inception of databases are constantly updated as new articles are digitized, searches were run without date limits. These databases represent major features of this paper: international health databases (PubMed, Embase, Web of Science), a psychiatry-specific database (PsycINFO), and an Africa-specific database (African Index Medicus). Africa Index Medicus is managed by the WHO, and is one of two major African health databases. The other is African Journals Online, which is not health specific. Our search strategy included terms related to the following ideas: (1) Africa (defined as the WHO Africa Region [25]), (2) detention or incarceration, and (3) mental health. Search syntax and controlled vocabulary words were modified for each database. Complete search terms for each database are in Additional file 1: Appendix S3.

Only primary research studies published in peer reviewed journals in English or French that met the following PICOS criteria were included. Participants included any people detained by the state, regardless of whether they had been sentenced to a crime. We excluded people formerly detained if data was collected after their release, and excluded people detained for immigration (similar to prior research on detained populations) [3]. We included studies that related to a mental illness as defined according to the DSM-V, but included studies that used other diagnostic criteria. Due to the scarcity of systematic research in this area, we included both qualitative and quantitative studies, including systematically collected data on policy, health systems, and conditions of detention, such as availability of physical or human resources, services, and the legal process. We included studies in the WHO Africa Region [25] and in any setting in which people were involuntarily held by the government for being accused or convicted of 


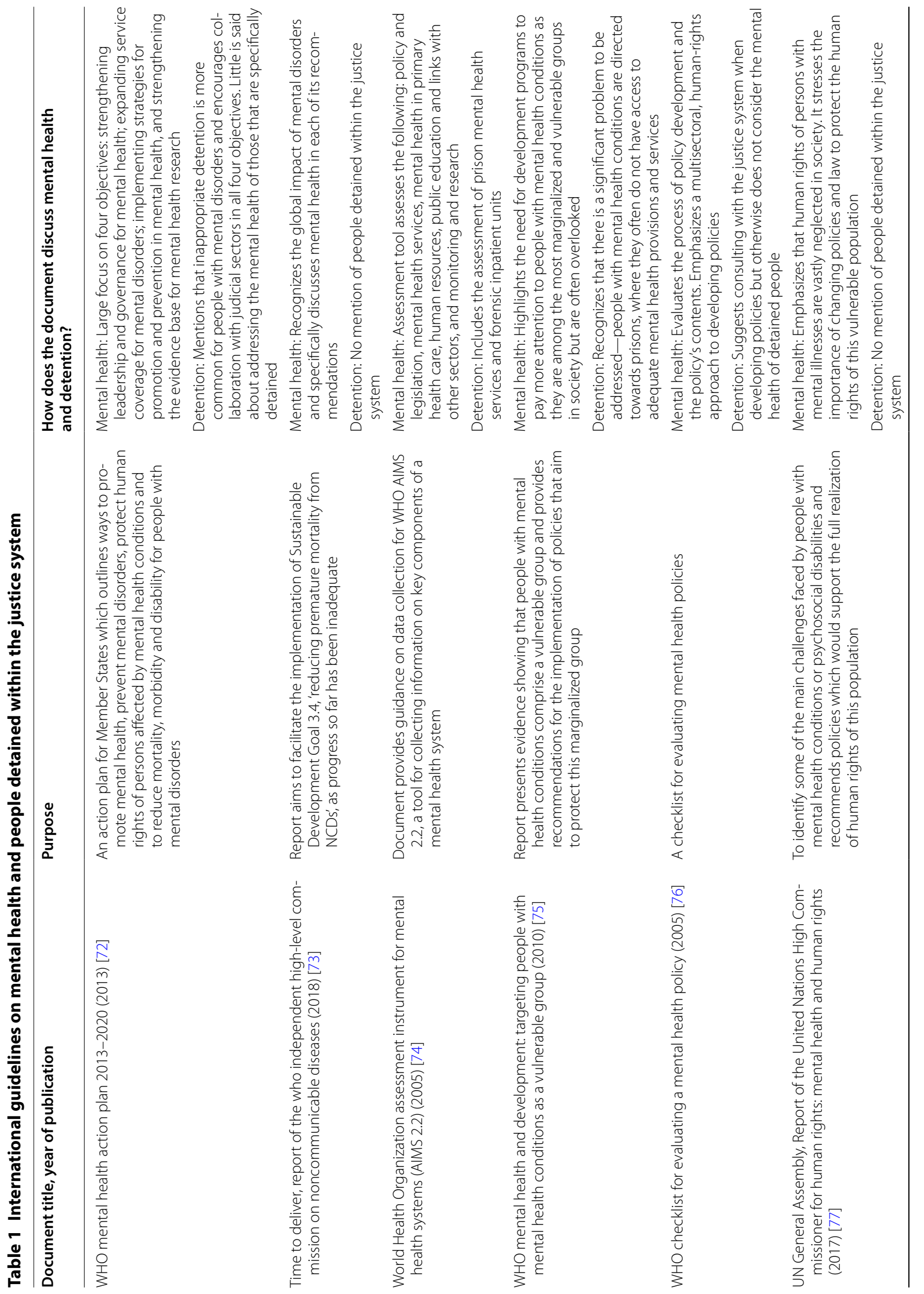


Lovett et al. Int J Mint Health Syst <wide> <wide> (2019) 13:31

Page 4 of 41

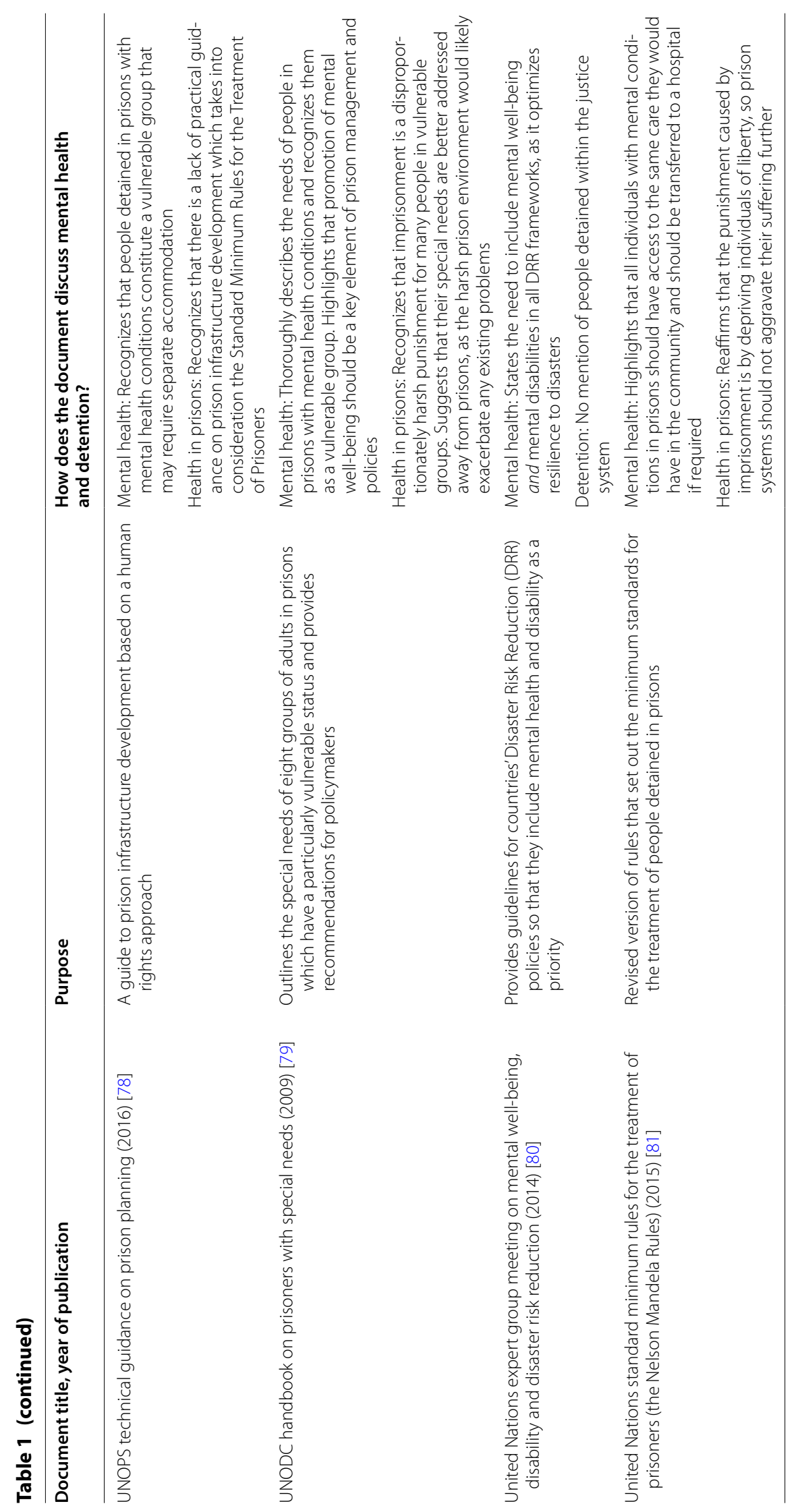


committing a crime. We excluded the settings of house arrest or non-forensic psychiatric hospitals in which people are involuntarily committed for disease alone.

\section{Data collection}

After the search was conducted and duplicates removed, two researchers (AL, HK-trained undergraduate students) separately screened the titles, abstracts, and full texts of studies to assess whether they met the inclusion criteria, reconciling differences between each step through discussion. A third reviewer $(\mathrm{HJ})$ acted as a tie breaker if the other screeners could not come to consensus. Following the full text screen, a backward search was conducted on all articles selected for inclusion. The results of the backward search were screened for inclusion following the same protocol as the initial search results (Fig. 1). If a paper identified could not be located using multiple university library systems, we attempted to contact the study author. If the author could not be contacted, we excluded this paper as it was not possible to access the full text. We did not contact authors to obtain missing data or details on methods.

\section{Risk of bias assessment}

Risk of bias was assessed using the following separate tools for each study type. Pre-post studies: Quality assessment tool for quantitative studies [26]; RCTs: Cochrane Collaboration's tool for assessing risk of bias [27]; qualitative: Critical Appraisal Skills Program (CASP) Checklist for Qualitative Studies [28]; prevalence: Prevalence Critical Appraisal Checklist [29]. We did not assess risk of bias for validation studies or structured reviews of health systems. The small number of these studies included in the review and the methodologic heterogeneity of structured reviews or descriptions of health systems make them difficult to compare with a single tool. We did not report a single overall risk of bias score for each study, as these scores typically involve arbitrarily weighting different domains of risk of bias [30]. Rather, we reported grouped studies into categories of high, medium, and low risk of bias based on the overall assessment generated by the methods assessment instruments. The methodological assessment was used to exclude studies that had either (1) insufficient information on methods or (2) a clear methodological flaw or inconsistency.

\section{Data extraction}

Data on study characteristics, sample characteristics, and study outcomes were extracted from each study (Tables 2, 3). Systematic reviews have the potential to elevate studies with poor ethics standards [31]; therefore, we extracted data on the presence or absence of documentation of an ethics committee review and informed consent procedures. If institution conditions or the laws or policy of the health or justice system were described, we extracted these descriptions and categorized the data into common findings (Additional file 1: Appendix S9).

\section{Data analysis}

Because of the heterogeneity of the study designs and outcomes, we conducted narrative analysis, as an overall meta-analysis was not possible. To facilitate comparison between like studies, we structured our presentation of results by study design.

\section{Meta-analysis}

The large number of prevalence studies enabled us to conduct a meta-analysis of the prevalence of mental disorders. Notably, this analysis was added post hoc, as we did not anticipate sufficient study design homogeneity for meta-analysis at the outset of the study. We generated pooled prevalence estimates with 95\% confidence intervals [32] for key disease categories: mental ill health (including measures of psychological distress or an unspecified mental disorder, often assessed using a screening tool, such as the General Health Questionnaire (GHQ) [33], but excluding results of disorder-specific instruments), mood disorders, psychotic disorders, and substance use. Heterogeneity between studies within each category was assessed using Chi squared and $\mathrm{I}^{2}$ ( $>50 \%$ is considered heterogeneous [34]). A random effects model was used to estimate the pooled prevalence, as all groups demonstrated significant heterogeneity. The random effects model weights included studies to account for both sample size and between-study variance with the between-study variance term dominating the weighting when studies are heterogeneous [35], as it assumes that studies come from different distributions. In order to identify sources of heterogeneity, we conducted subgroup analysis, grouping according to youth and adults; then, within studies of adults, location (prison or forensic ward) and data collection method (instrument or clinical record). In the subgroup analysis, we determined the pooled prevalence for each subgroup and its heterogeneity. Statistical analysis was done using STATA/ SE 15.0 [36]. Additional details of the data extraction and statistical analysis are shown in Additional file 1: Appendices S4, S6 and S7.

\section{Results}

Search results

After removing duplicates, our search yielded 1240 results in the initial database search, of which 73 met inclusion criteria. We excluded three studies based on the methods screen results and added eight from a backward search. We were not able to access the texts or abstracts 
of three studies from the backward search title results because they were not available for loan from multiple university library systems. A third researcher $(\mathrm{HJ})$ reviewed 39 full texts about which the other two reviewers could not reach agreement and chose to include 12 of them (31\%). We added two articles identified through expert consultation. This yielded 80 papers for data extraction (Fig. 1), including 17 papers that were written on the same sample and as part of the same study as another paper in our dataset, but with different methods details or outcomes reported. In this review, the terms "independent studies" or "samples" refer to the number of independent studies, and the term "papers" refers to all papers in the dataset. There were 70 independent studies in our data. All papers are described in Tables 2 and 3.

\section{Study characteristics}

Prevalence was the dominant study type (67\%), with small numbers of other study types including ten nonprevalence cross-sectional studies, four qualitative studies, four tool validations, two structured health systems reviews, two pre-post studies, and one randomized control trial. The majority of studies took place in either Nigeria (30;43\%) or South Africa (21;30\%). Only five of 23 non-prevalence studies took place outside of Nigeria or South Africa: two health systems studies in Zimbabwe, one qualitative study in Zambia, one cross-sectional study in Rwanda, and one international comparative study. Sixty-nine percent of studies collected primary data, or data resulting from diagnostic or screening tools, and $37 \%$ collected secondary data, defined as data extracted from available records. Four studies collected both primary and secondary data. Thirty-two studies (46\%) examined prison settings, of which all but one study collected primary data. Twenty-six studies (37\%) examined forensic hospital settings (included only if they were settings in which a justice-involved population was detained). Among these, $88 \%$ were based on secondary data, and the majority took place in either South Africa (54\%), Nigeria (15\%), or Zimbabwe (12\%). Nine studies (13\%) examined youth institutions, of which all were conducted in Nigeria. Three studies (4\%), two conducted in Zimbabwe and one international, examined the mental health system.

\section{Risk of bias}

Most of the papers assessed fell into the low (36; 49\%) or medium $(33 ; 45 \%)$ methods risk of bias categories. A number of medium risk papers were given this judgement based on lack of detail in methods reporting, meaning that it was unclear if the studies had high risk of bias or if methods were not reported well. About half of the medium risk papers reported diagnoses from secondary psychiatric records (17), for which it was often unclear how the diagnoses were made, who made them, or if they were based on standard criteria, such as the ICD. Of the 57 psychiatric prevalence papers, $44 \%$ (25) fell into low risk, $47 \%$ (27) fell into medium, and 9\% (5) fell into high risk of bias categories. All "high risk" studies were prevalence papers. See Additional file 1: Appendix S5 for results of the methodologic review of prevalence studies, which includes evaluation of sampling technique using the Prevalence Critical Appraisal Checklist.

\section{Data collection method}

Most studies used a census sampling strategy (42;60\%), followed by random $(18 ; 26 \%)$, purposive $(8 ; 11 \%)$, not stated $(4 ; 5.7 \%)$, mixed sampling $(3 ; 4.3 \%)$, and convenience sampling (1, 1.4\%). Of all psychiatric prevalence papers, $60 \%$ used validated instruments for primary data collection, while the rest extracted data from secondary psychiatric records.

\section{Participants}

In $69 \%$ of samples, more than $85 \%$ of participants were male. In 10 samples, "male" was an inclusion criterion. The mean age of participants among samples listing mean age was 28.8 , and there was a broad distribution of sample size, from $18 \%$ of participant samples with 50 people or less (including all three interventions), to 13\% with over 400 participants. In $36 \%$ of samples, the majority of participants had not been convicted of any crime (the majority of participants were either awaiting trial or detained without trial). In another $41 \%$ of samples, trial status was not stated or unclear, or participants were justice-involved youth (in which trial status was ambiguous). In only $20 \%$ of samples, the majority of participants had been convicted.

\section{Ethics characteristics}

Of all papers, 35\% neither documented ethics committee approval nor described an informed consent procedure, and $41 \%$ described both. Ten percent reported ethics committee approval but not informed consent, and 14\% reported informed consent but not ethics committee approval.

\section{Outcomes}

To facilitate comparison between like outcomes, we have presented study outcomes by study design: prevalence, non-prevalence cross-sectional, intervention testing, qualitative, structured health system review, and tool validation. Of the 57 prevalence papers reporting mental health diagnoses or screening results, 44 (77\%) reported diagnoses of psychiatric conditions, of which 14 papers also used instruments designed to screen for psychiatric 


Database search results
Pubmed: 474
Embase: 540
PsyclNFO: 386
Web of Science: 499
African Index Medicus: 12
Total: 1911
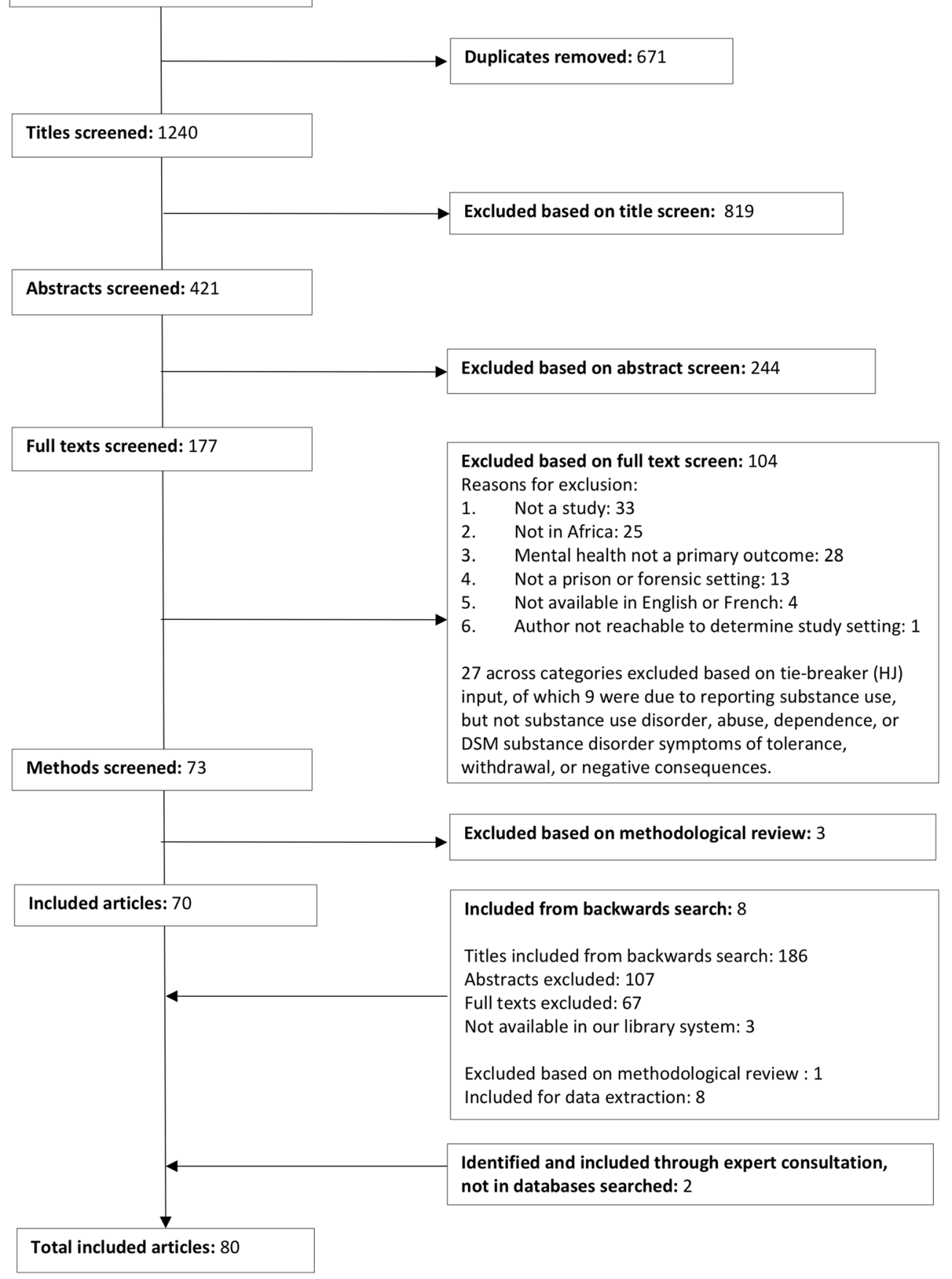

Excluded based on methodological review: 3

Included from backwards search: 8

Titles included from backwards search: 186 Abstracts excluded: 107

Full texts excluded: 67

Not available in our library system: 3

Excluded based on methodological review : 1 Included for data extraction: 8

Identified and included through expert consultation, not in databases searched: 2

Fig. 1 Flowchart 


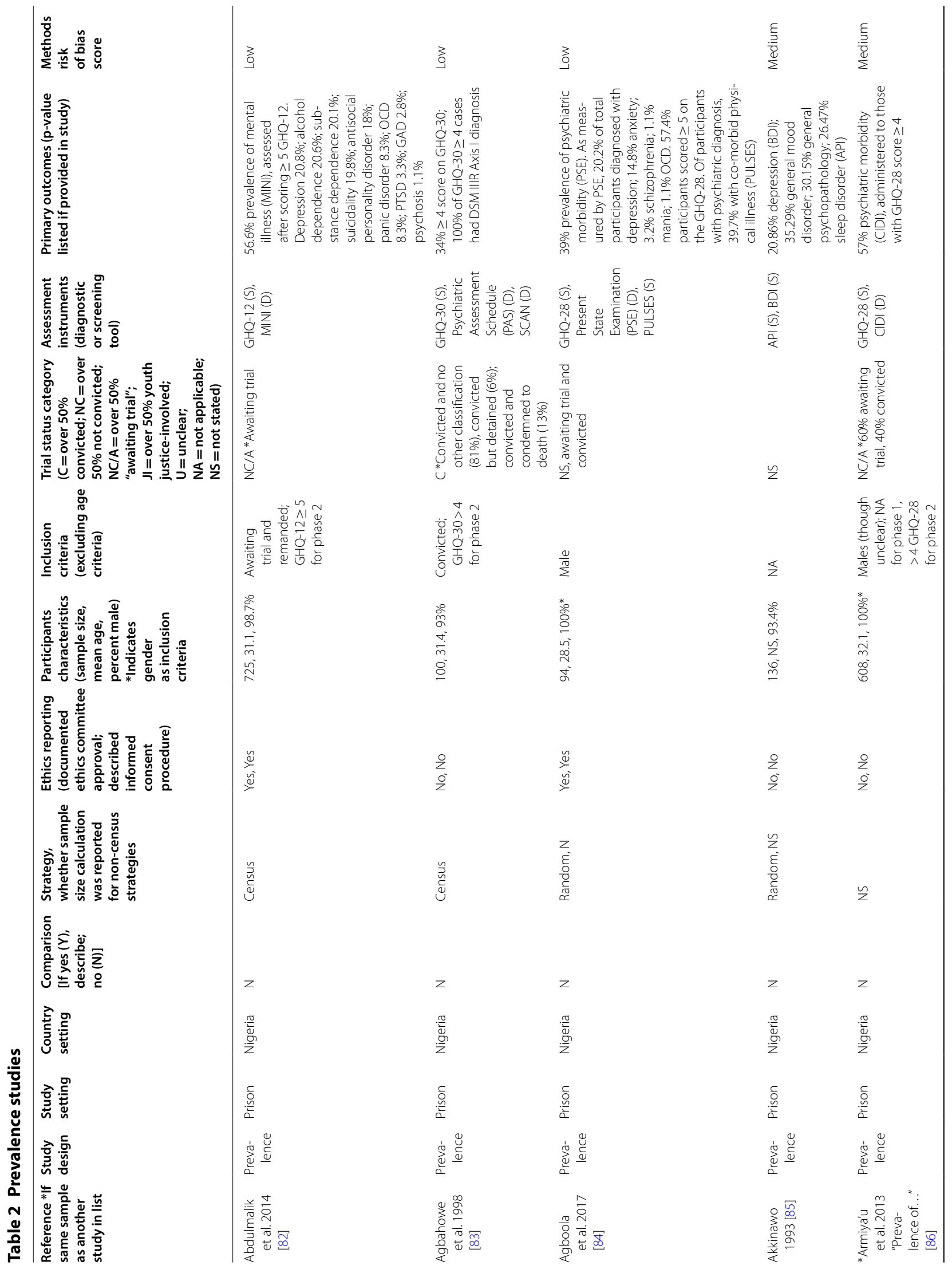




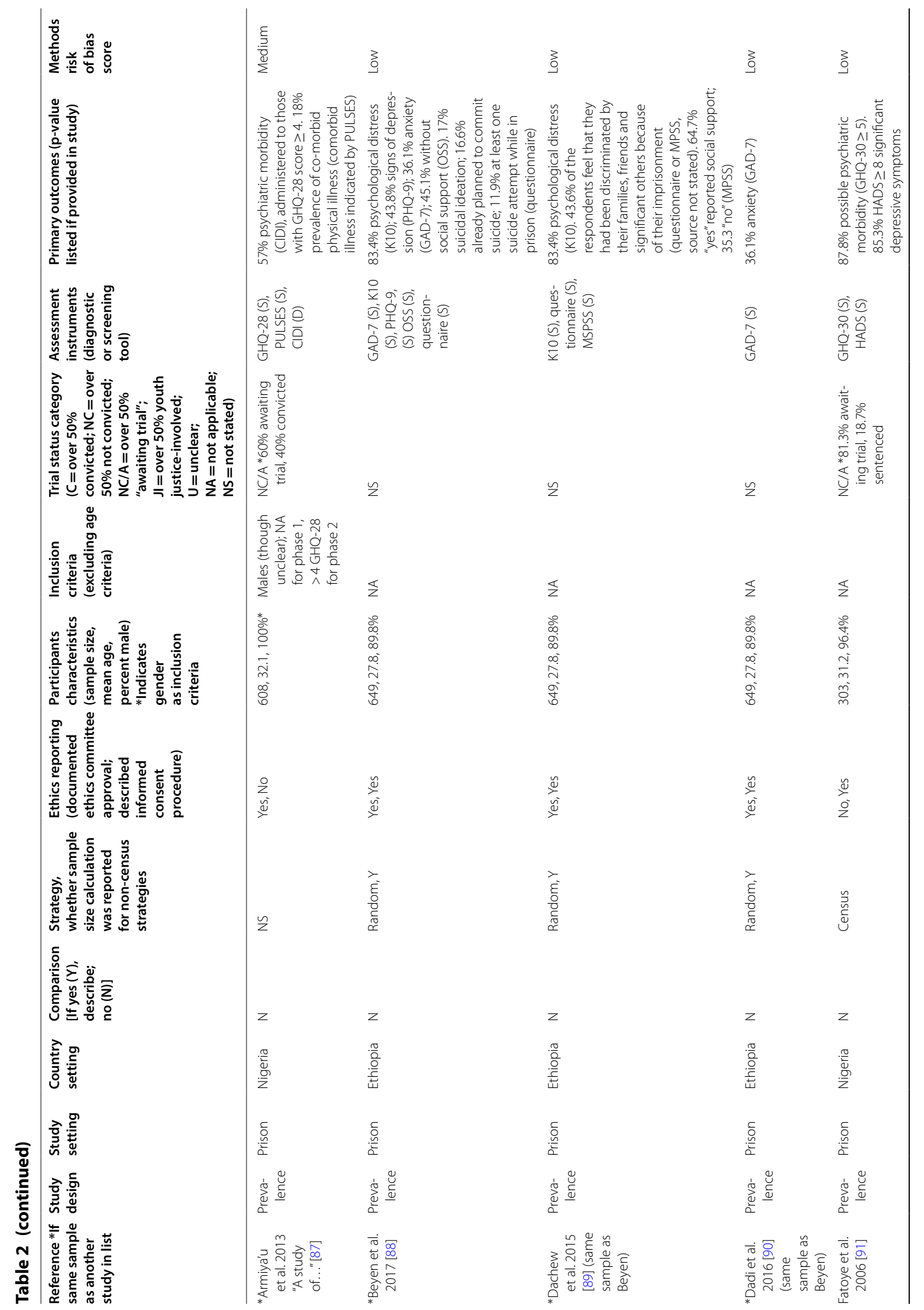




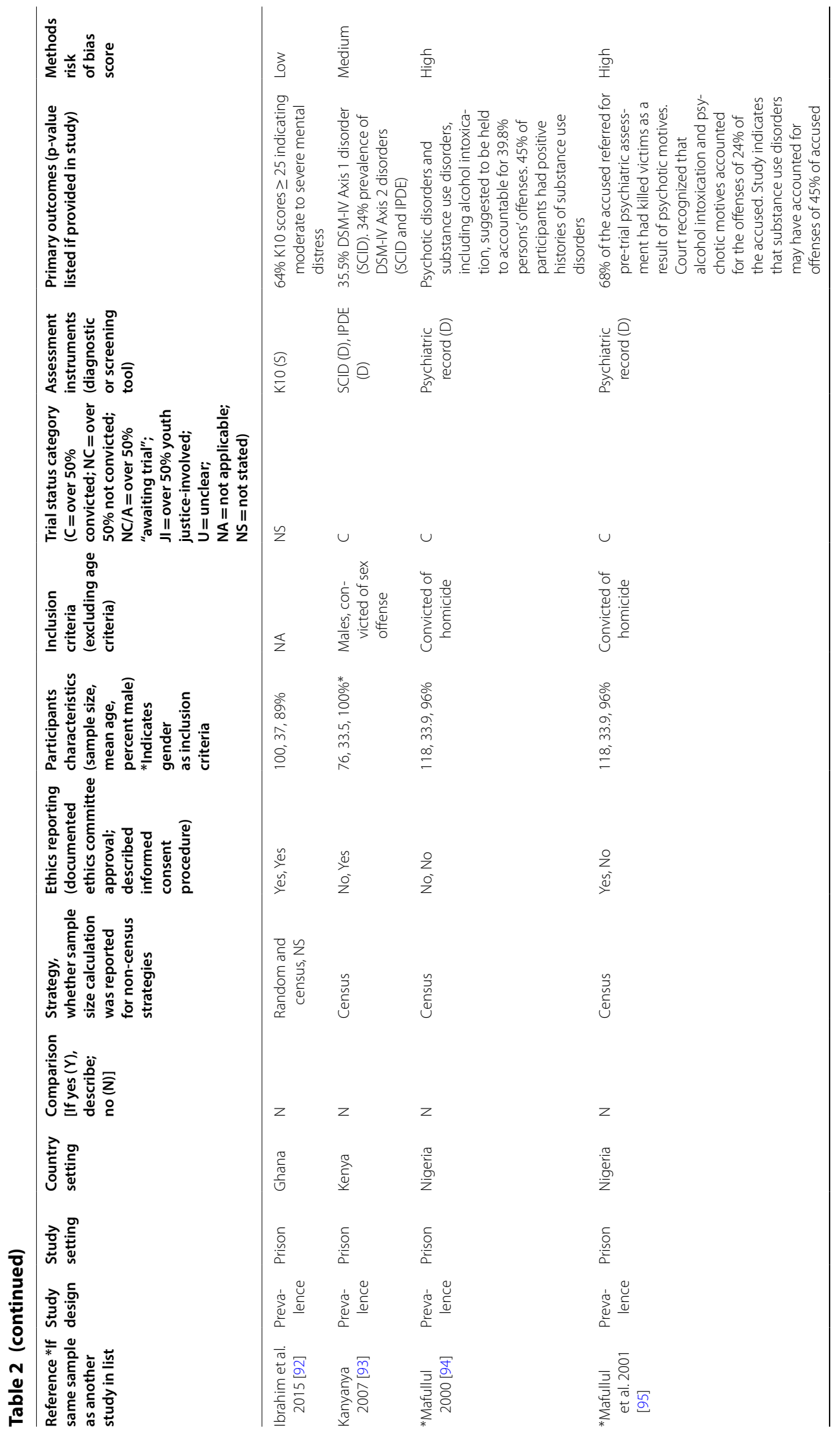




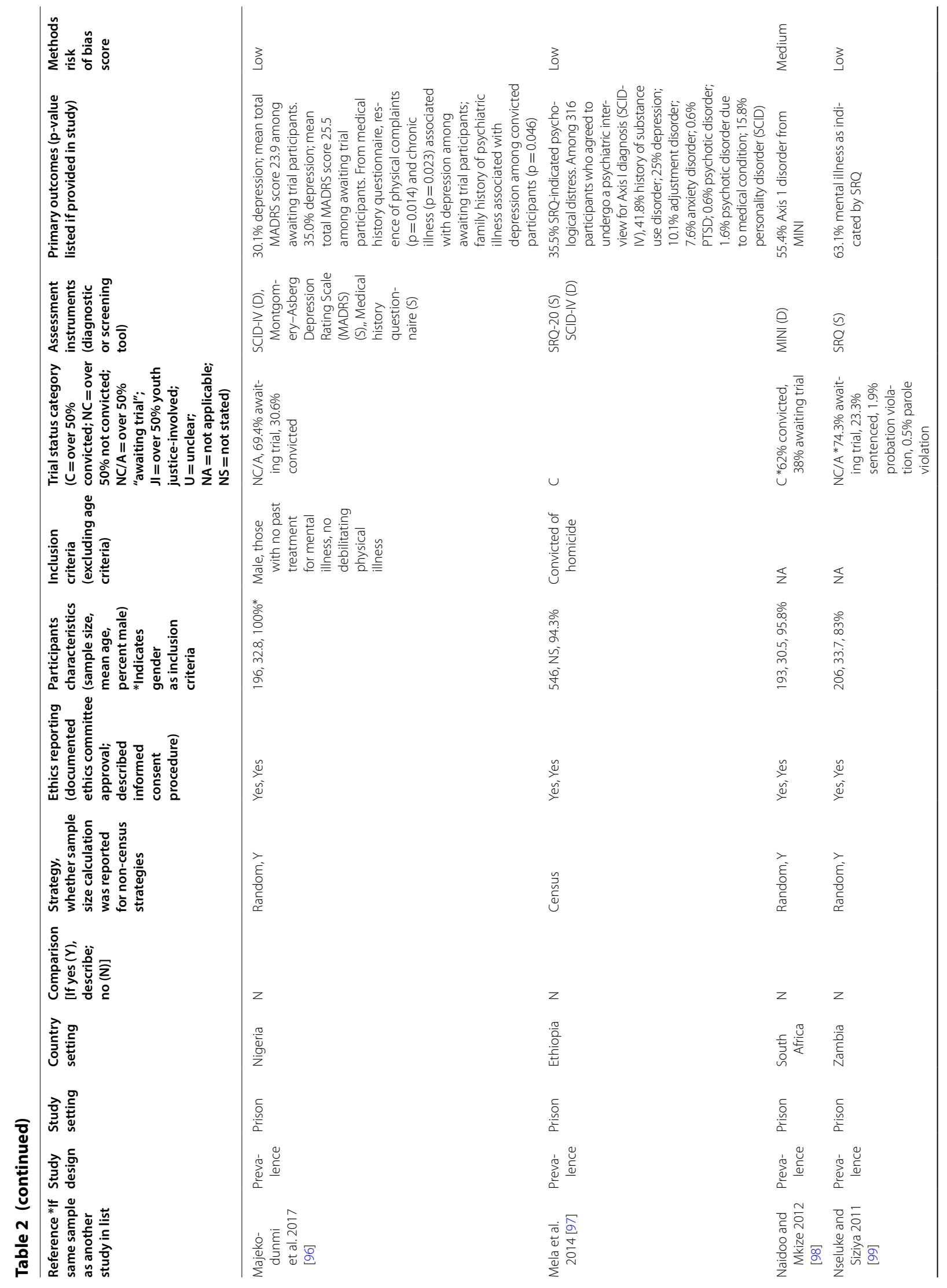




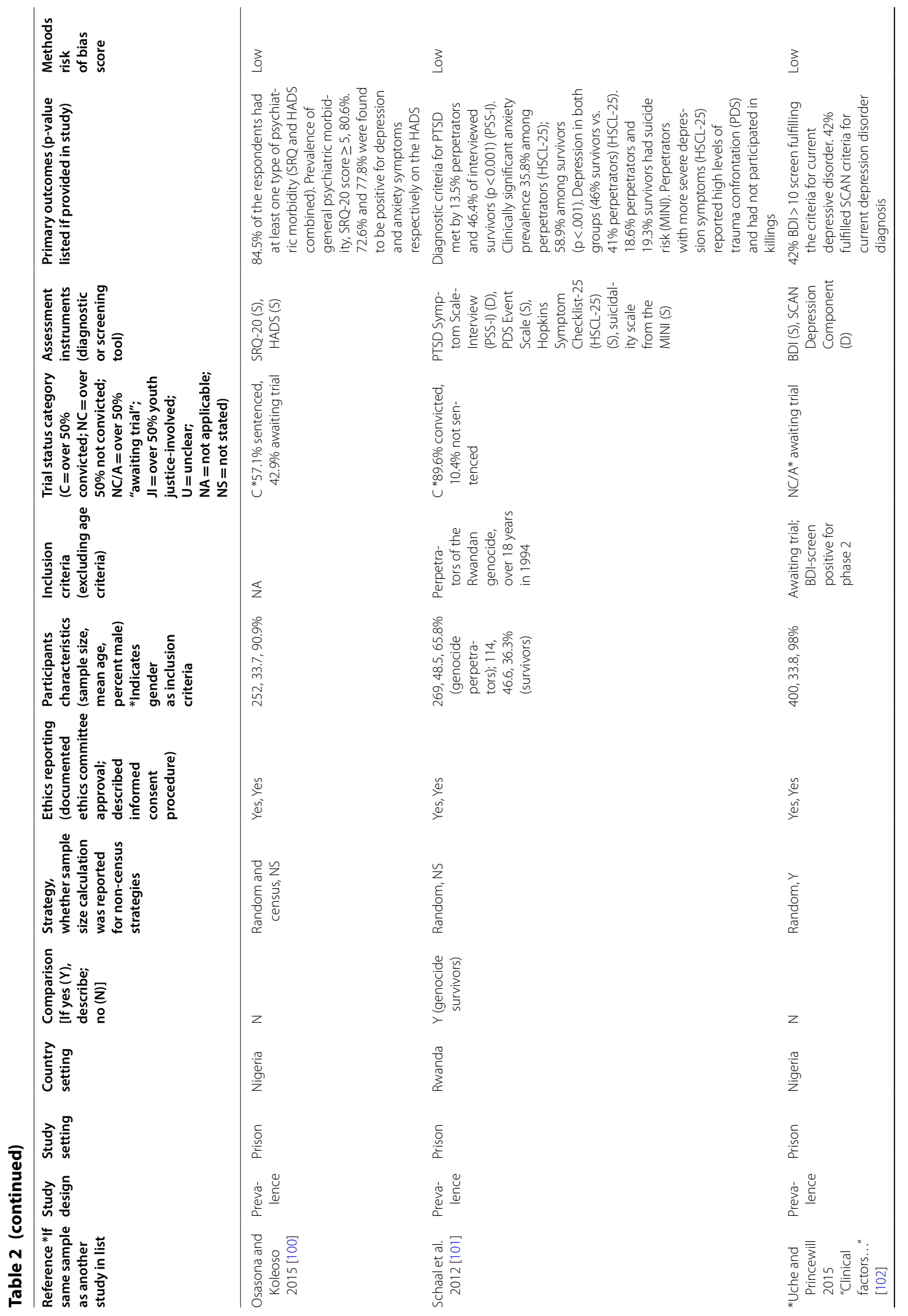




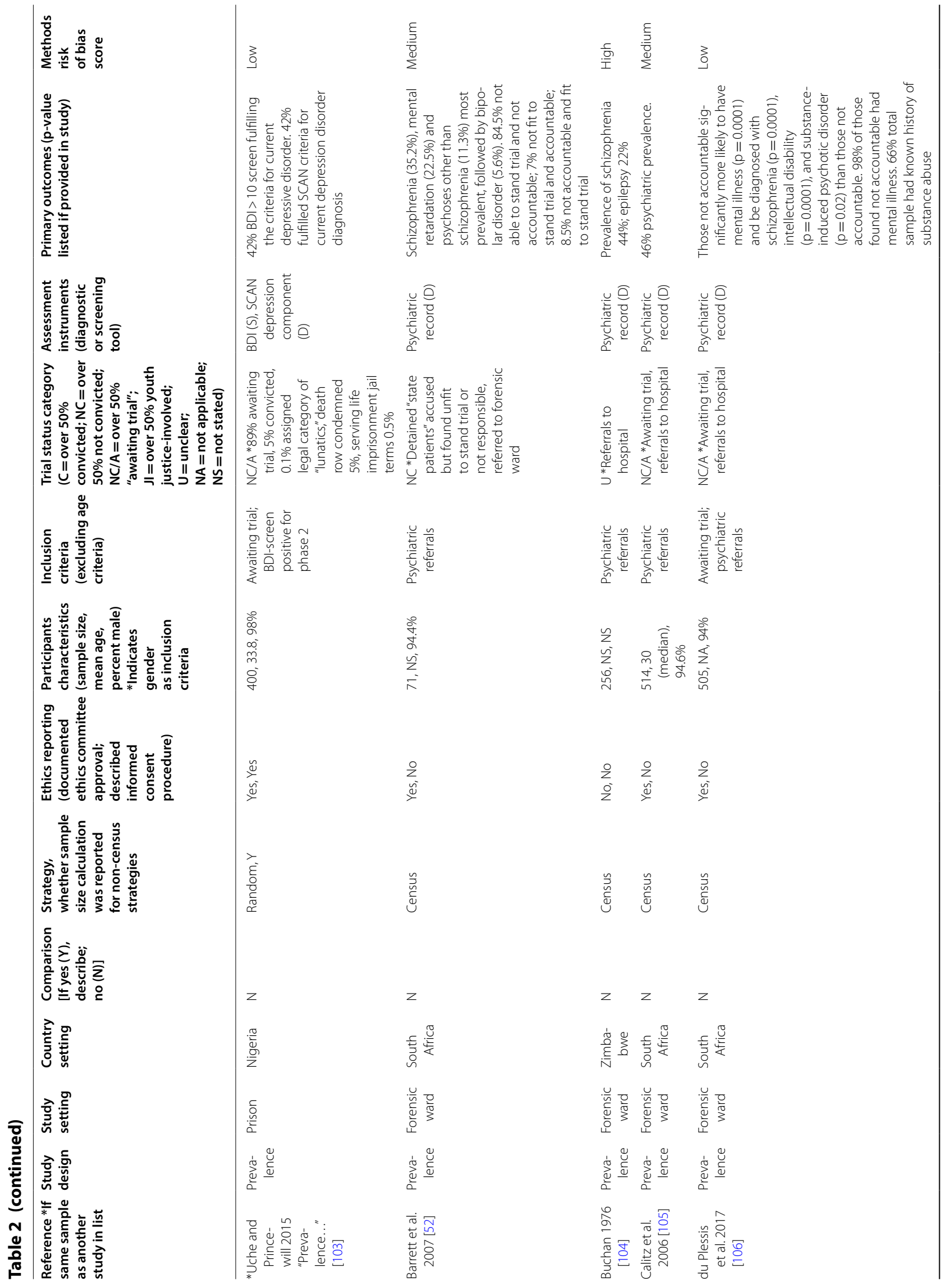




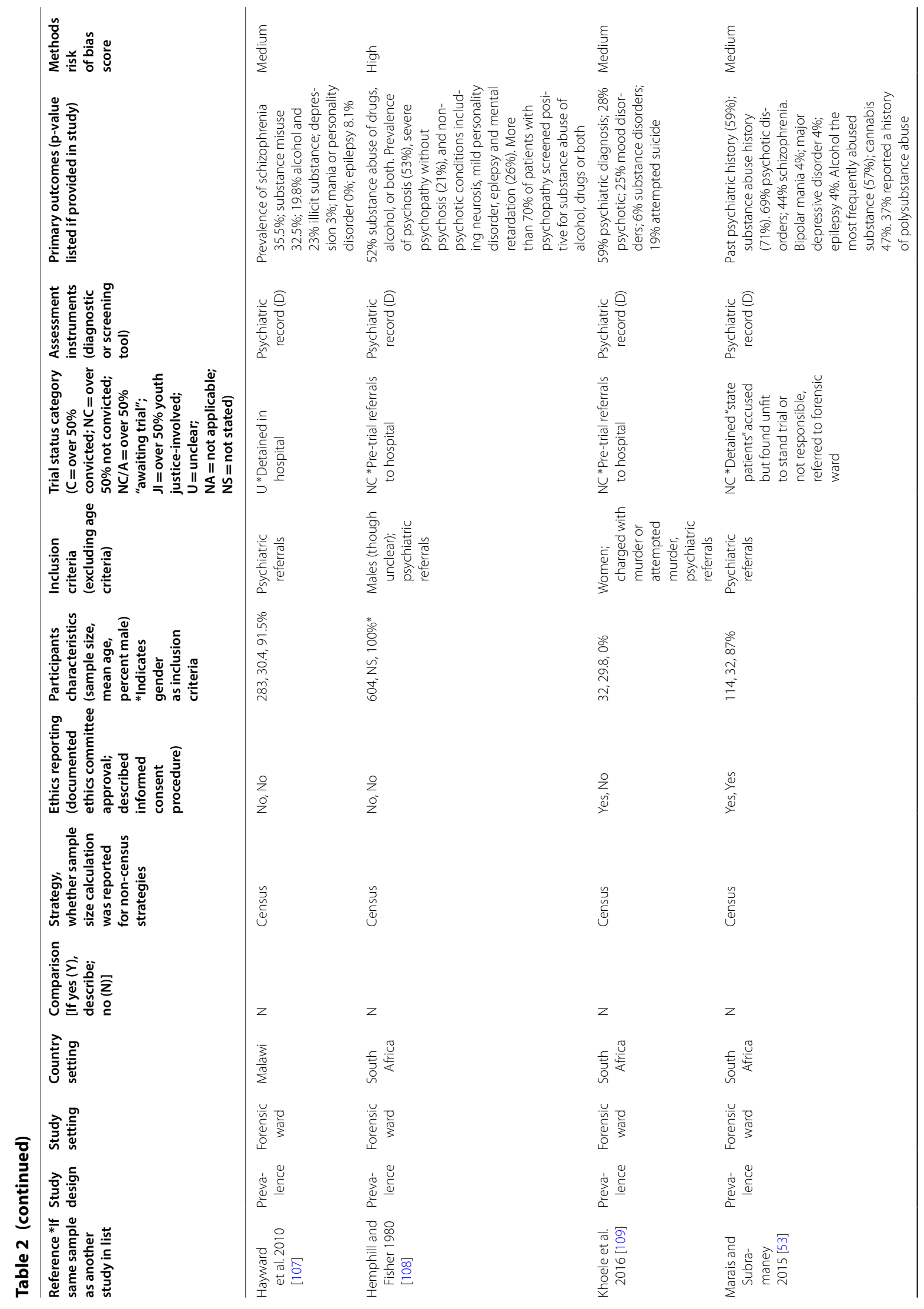




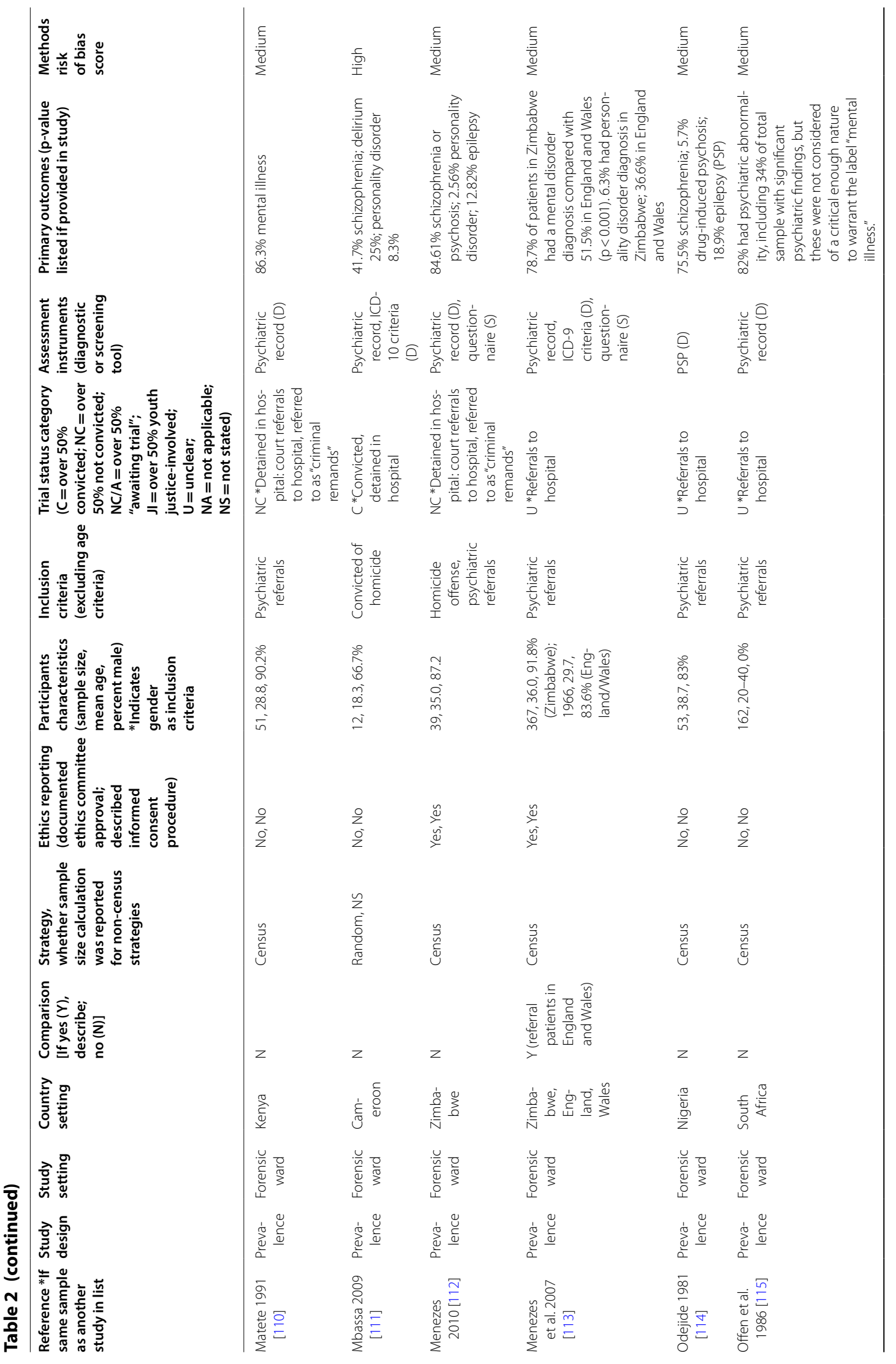




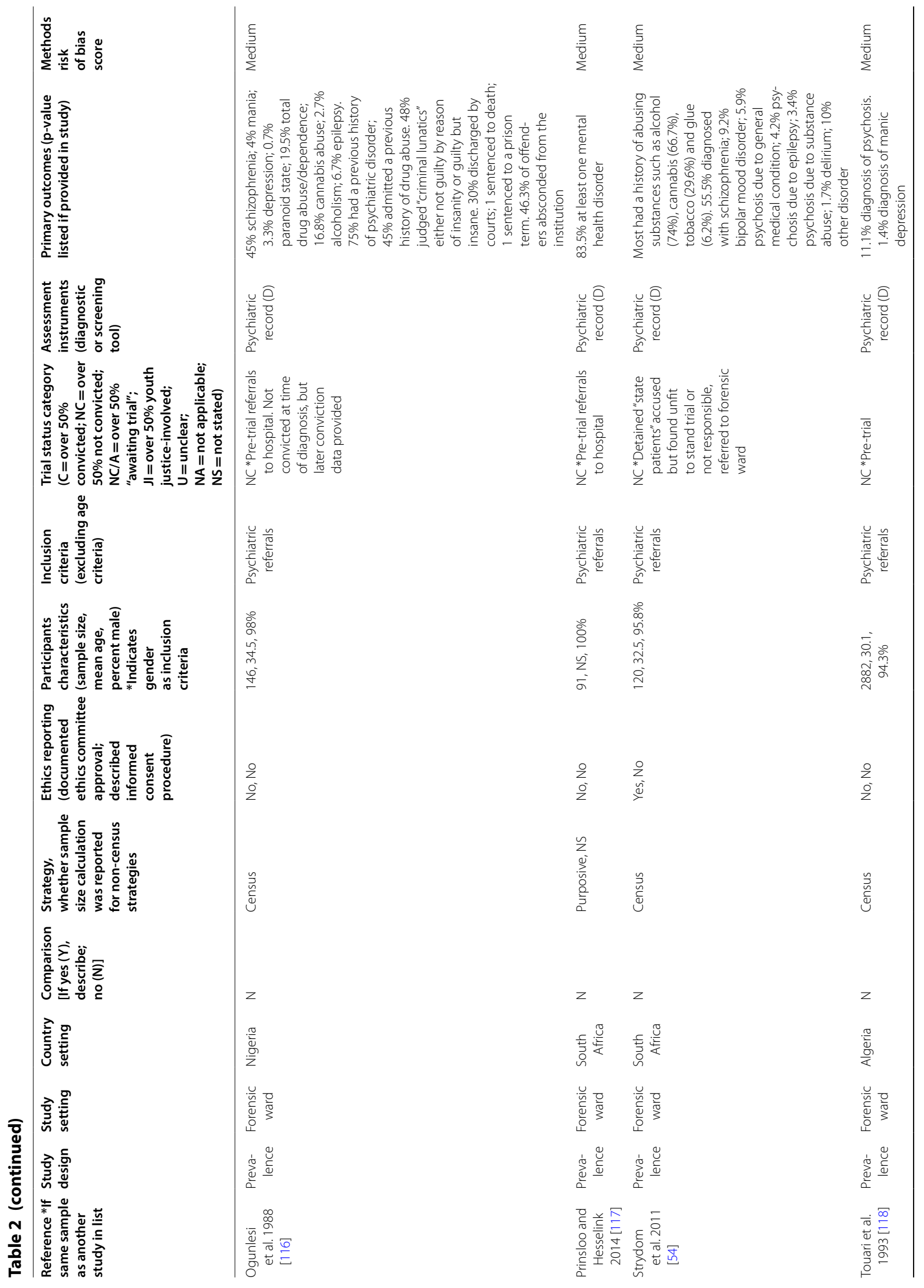




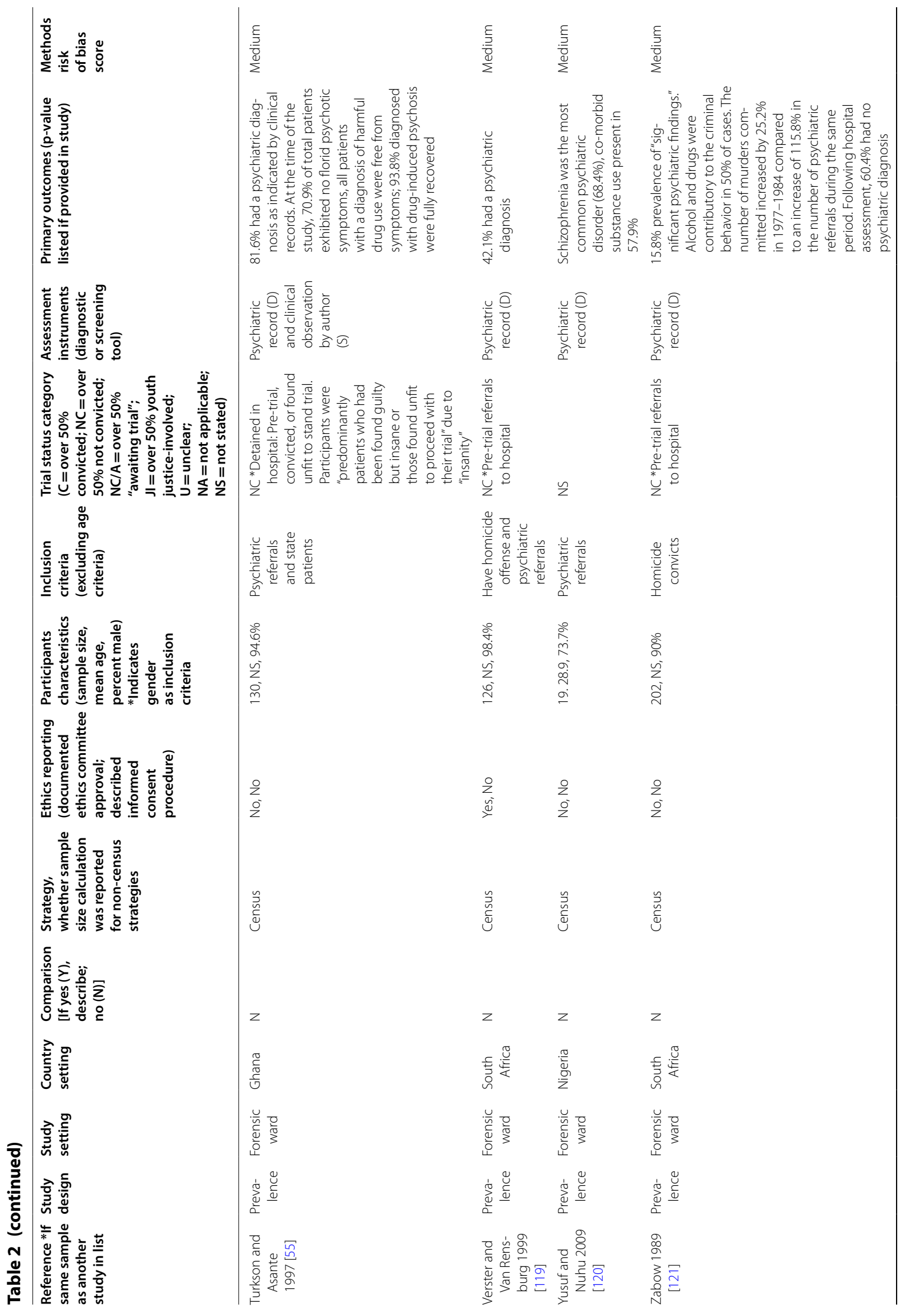




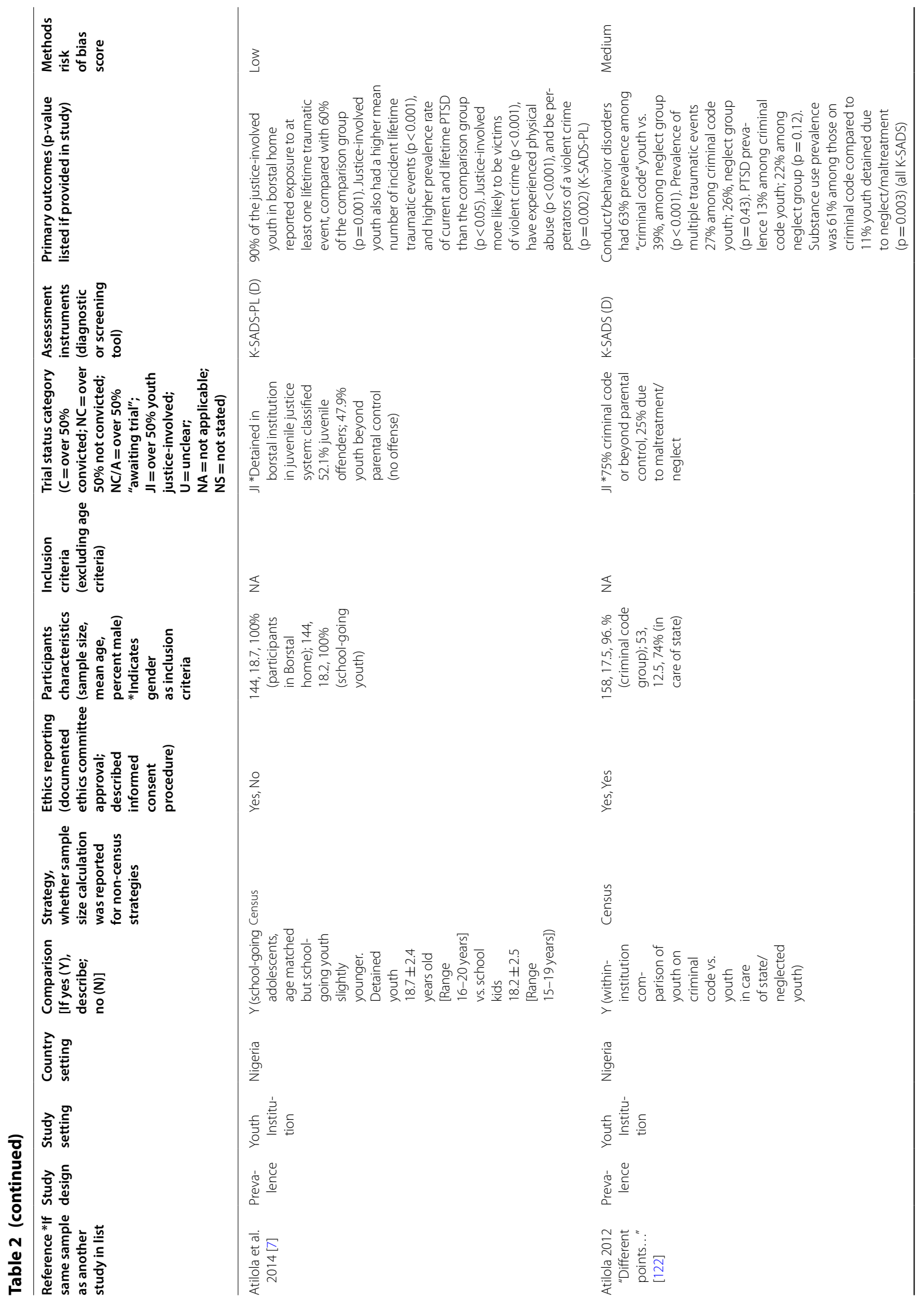




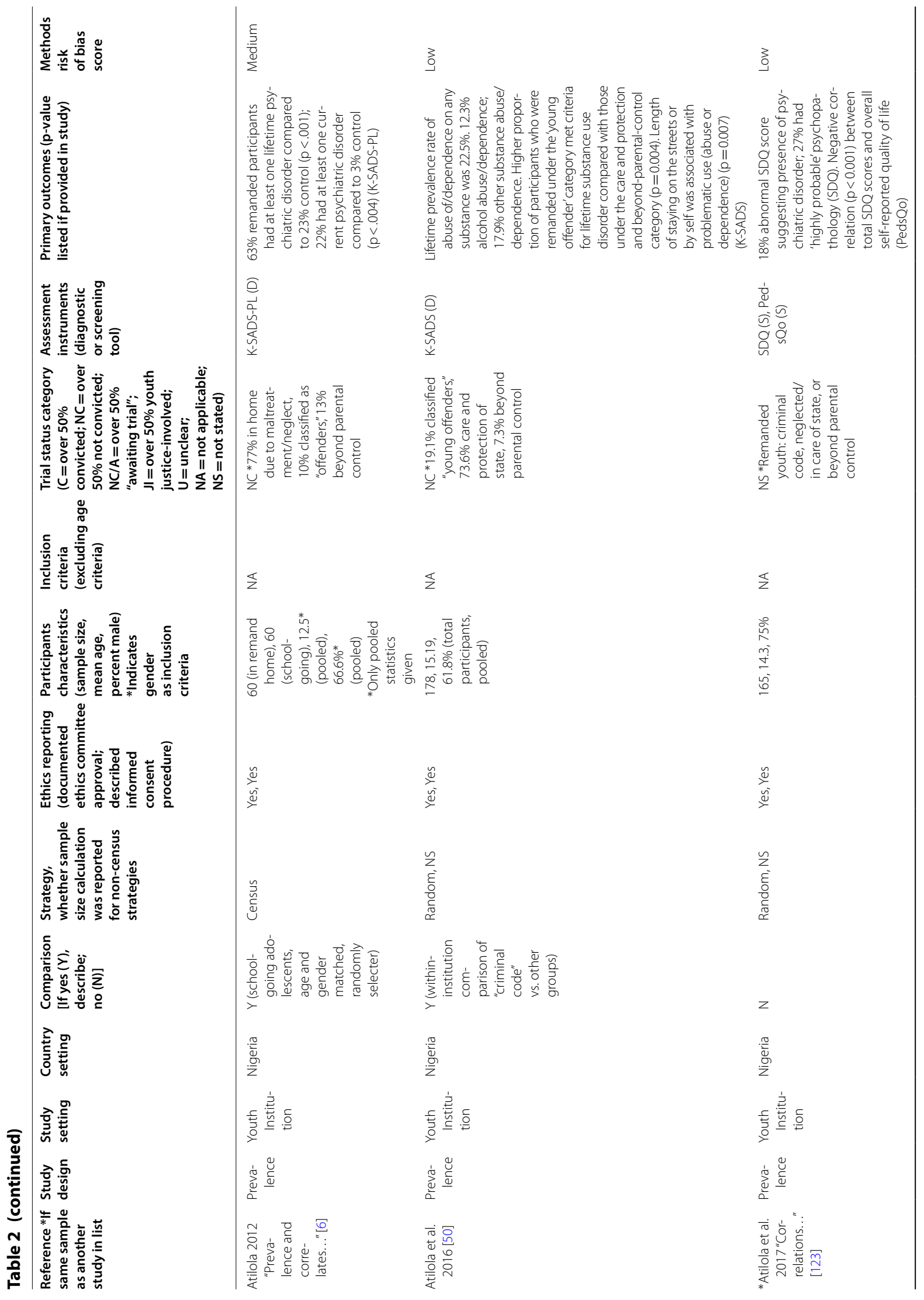




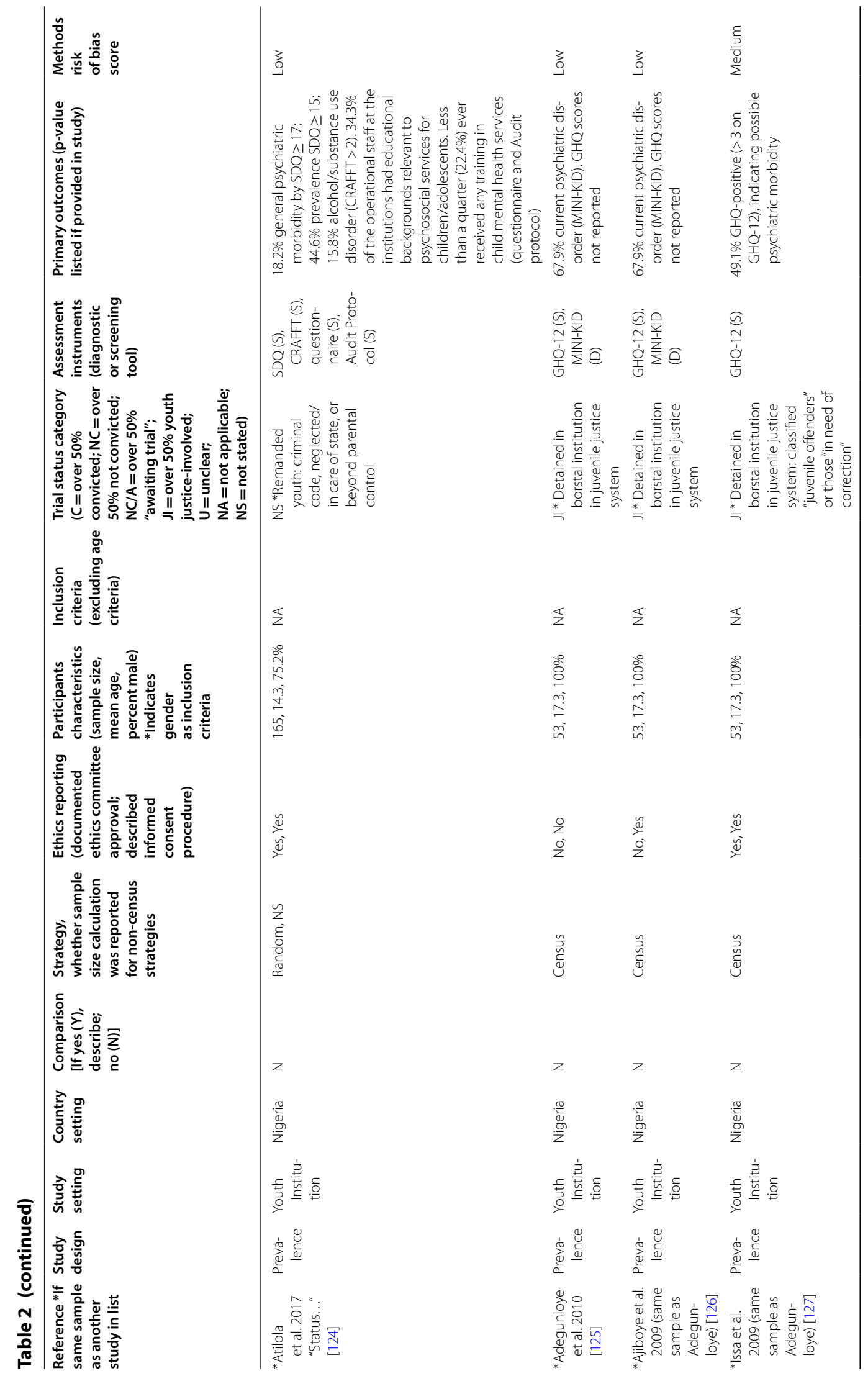




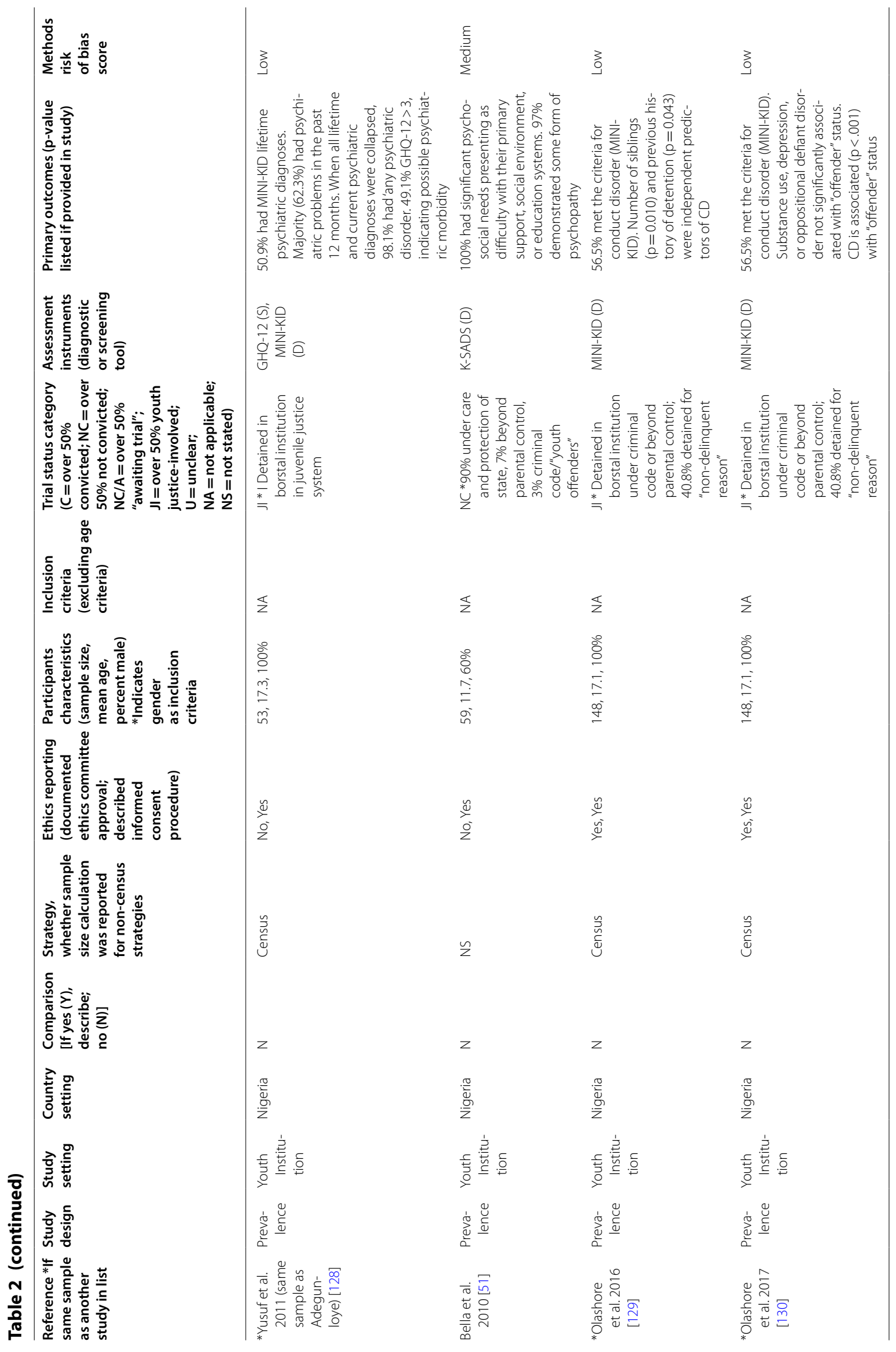




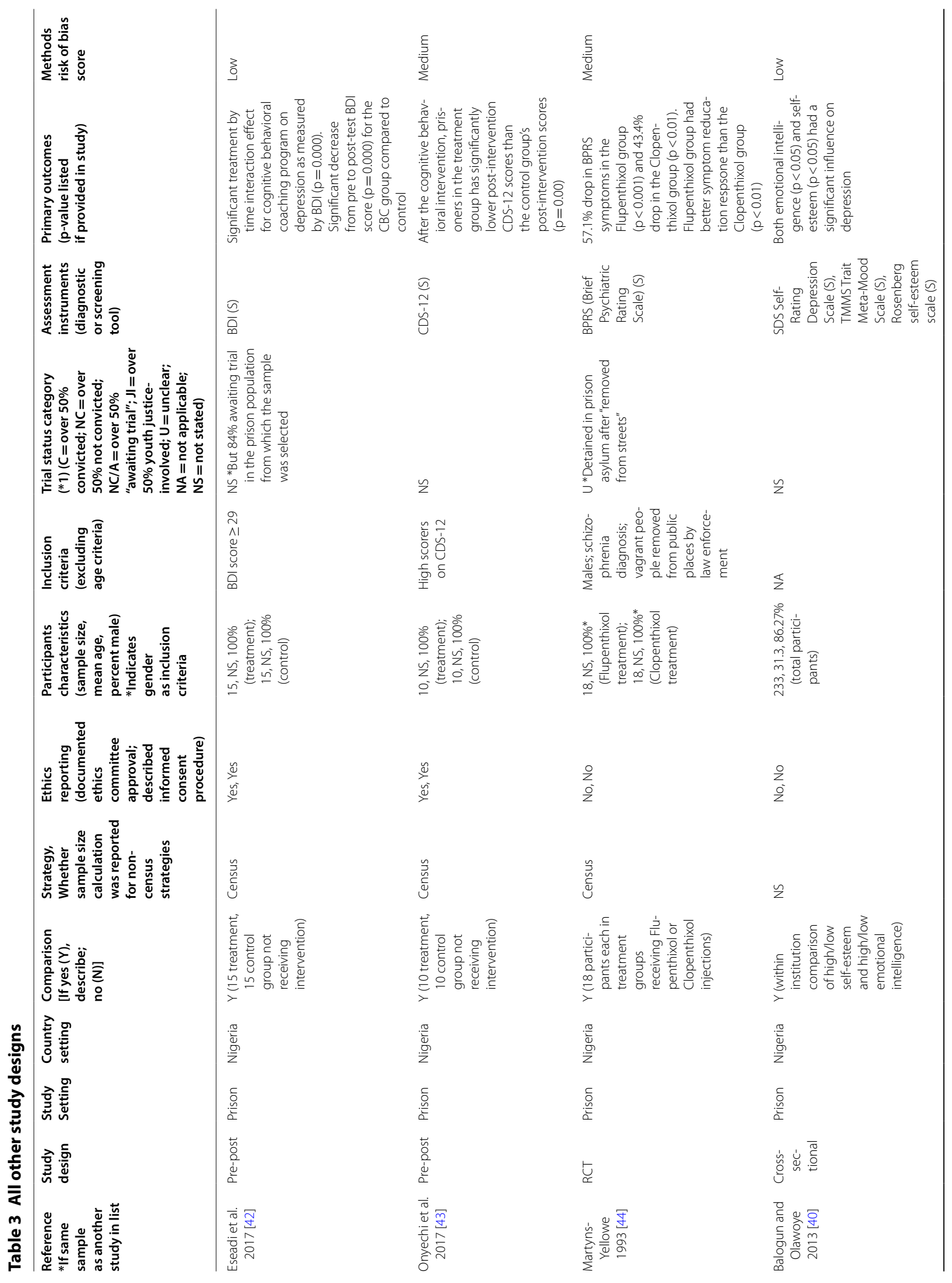




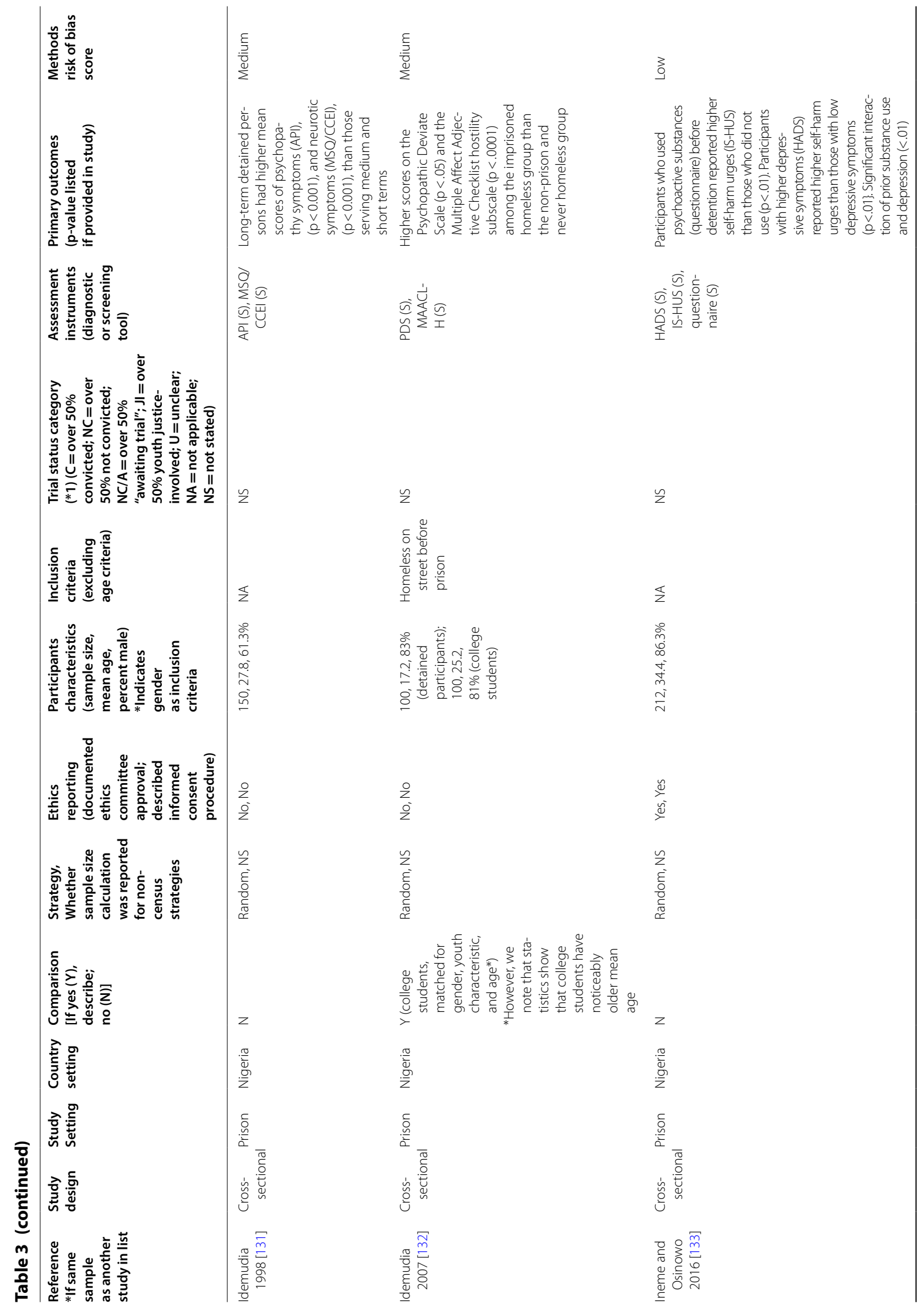




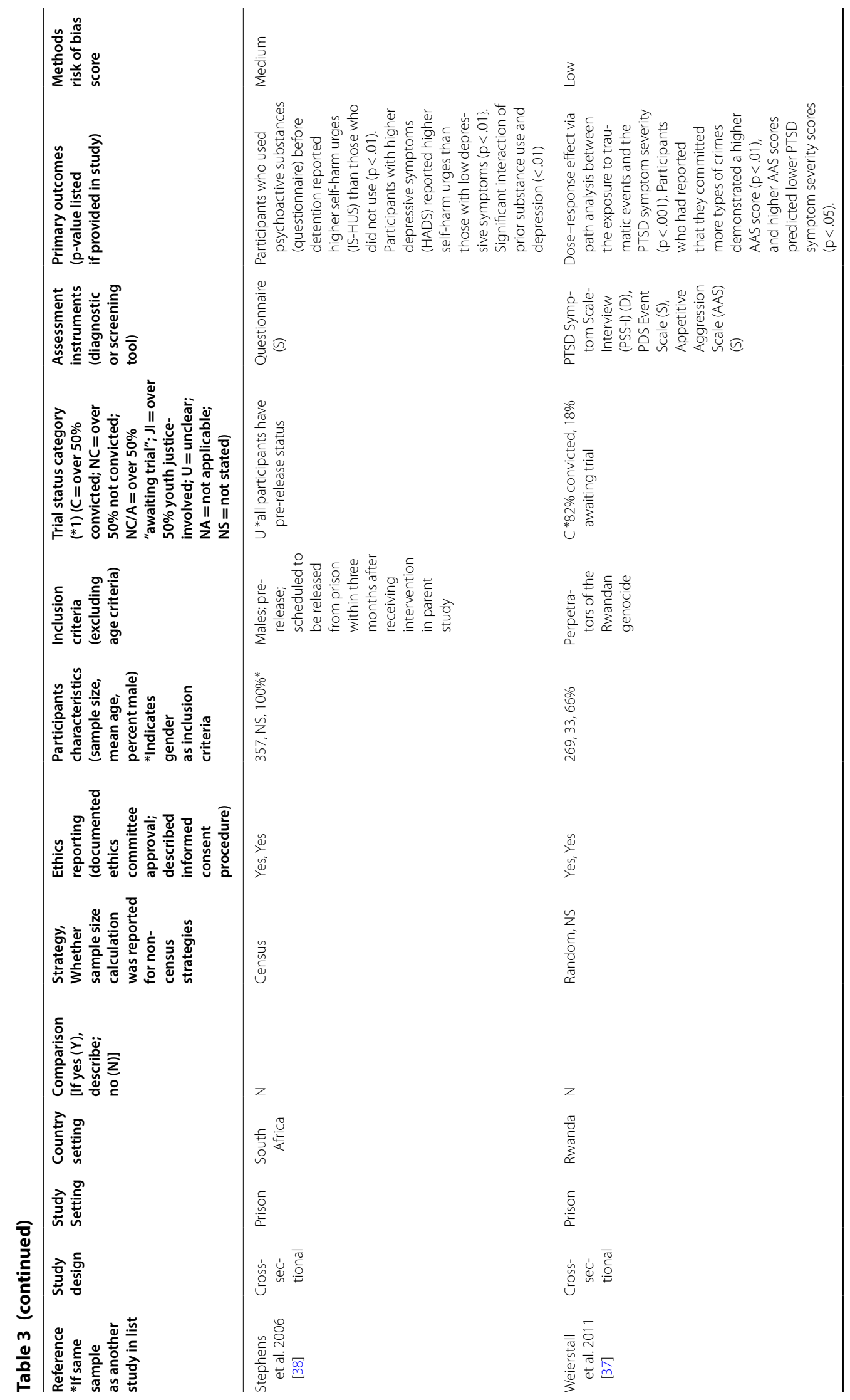




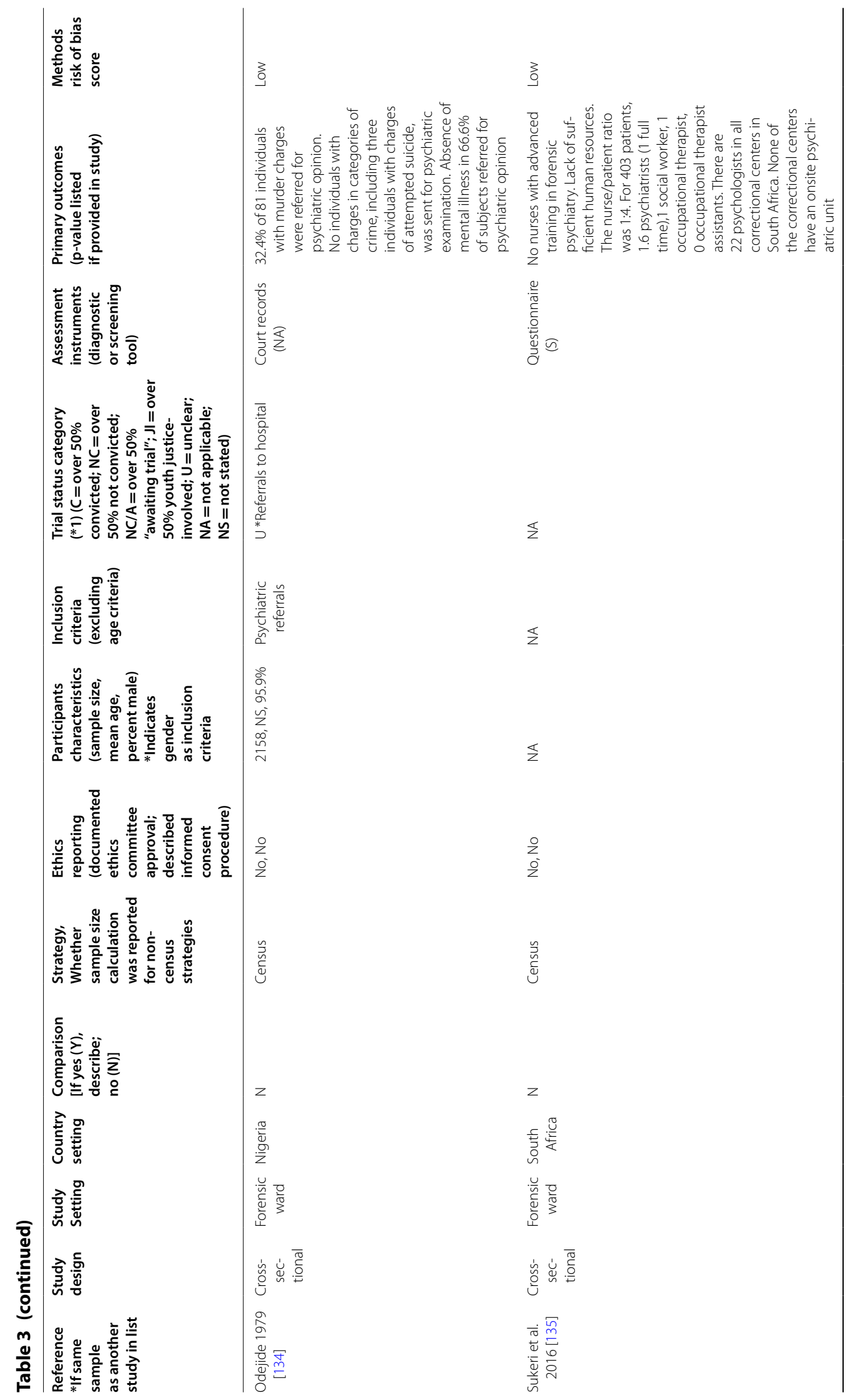




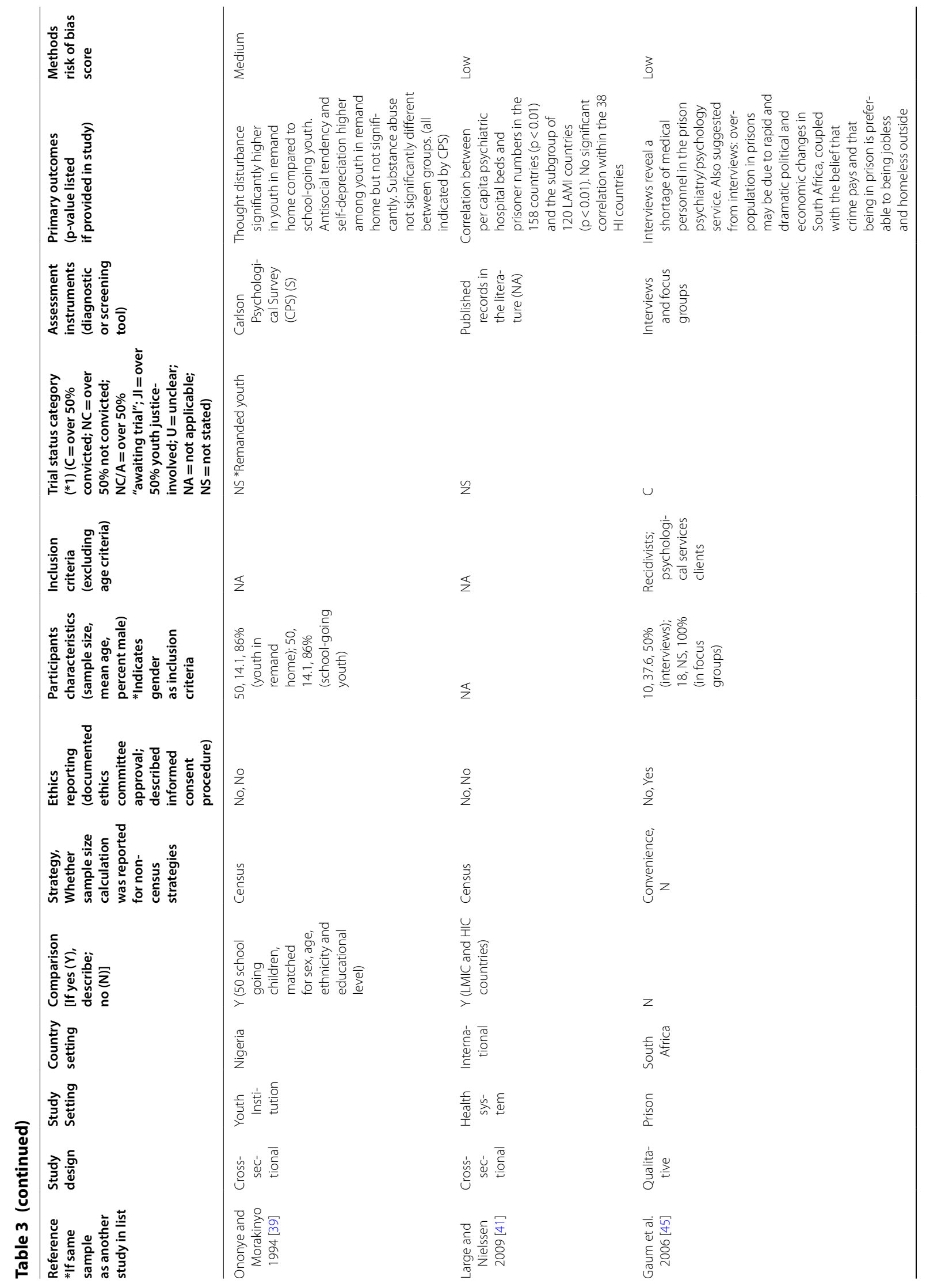




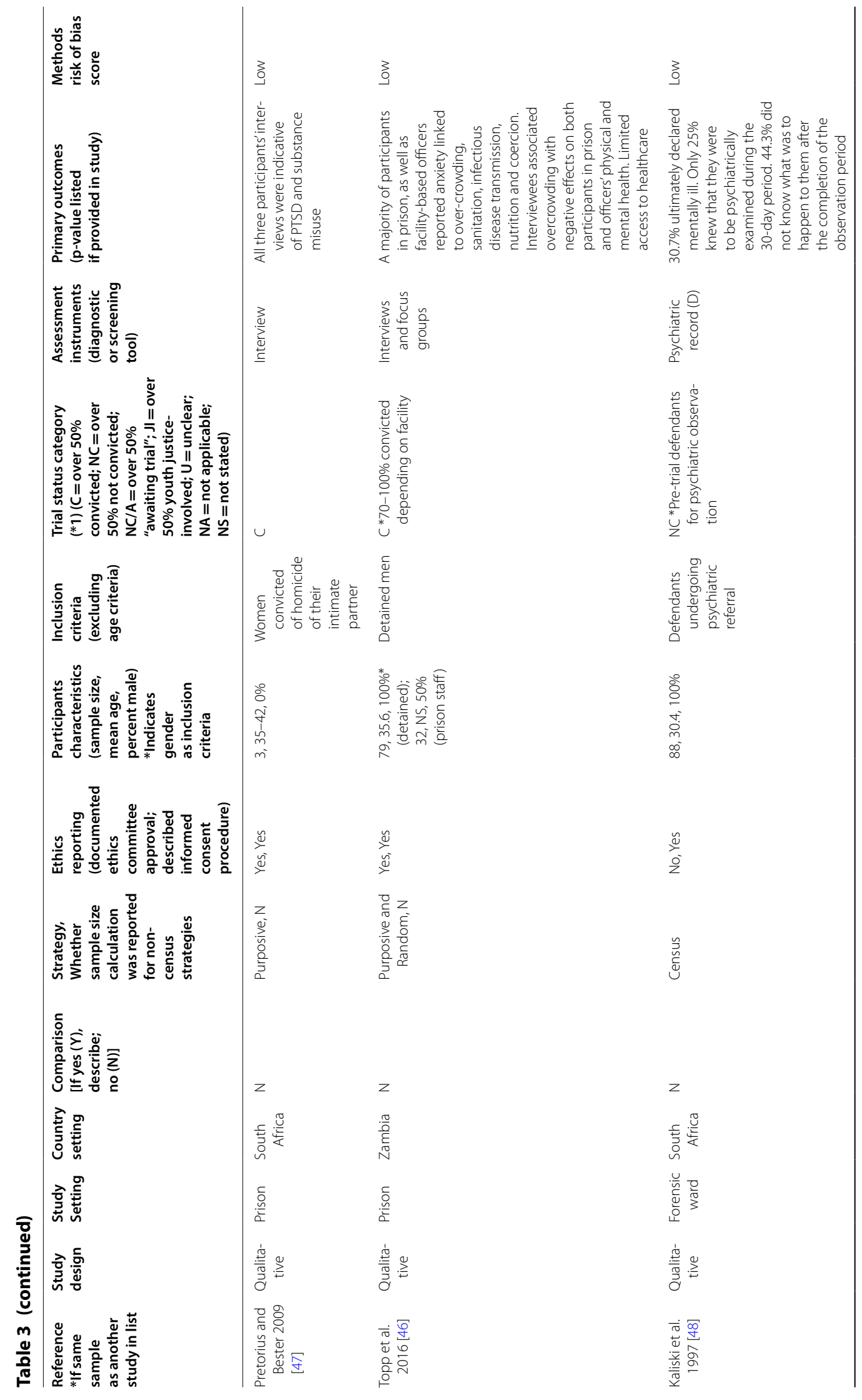




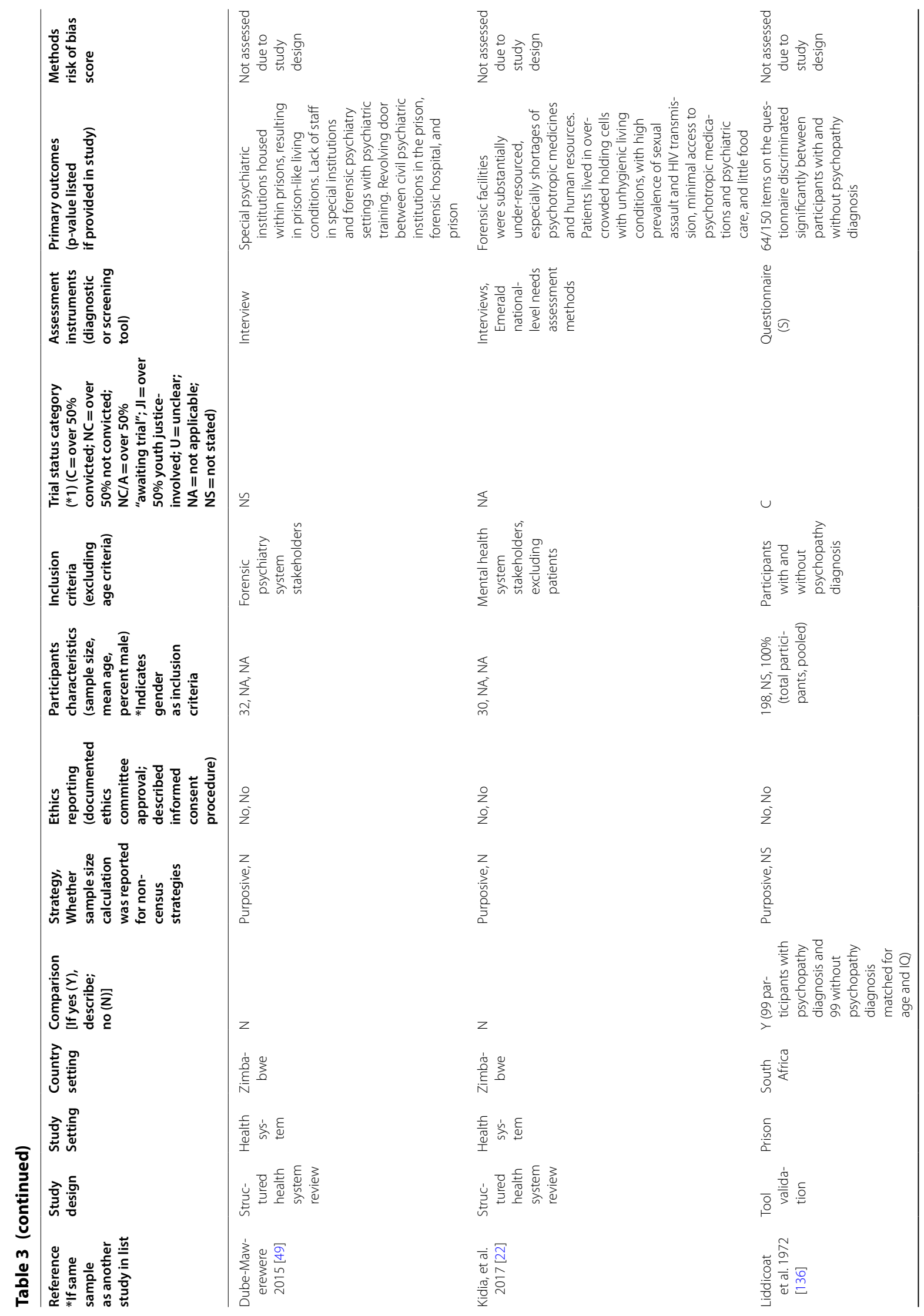




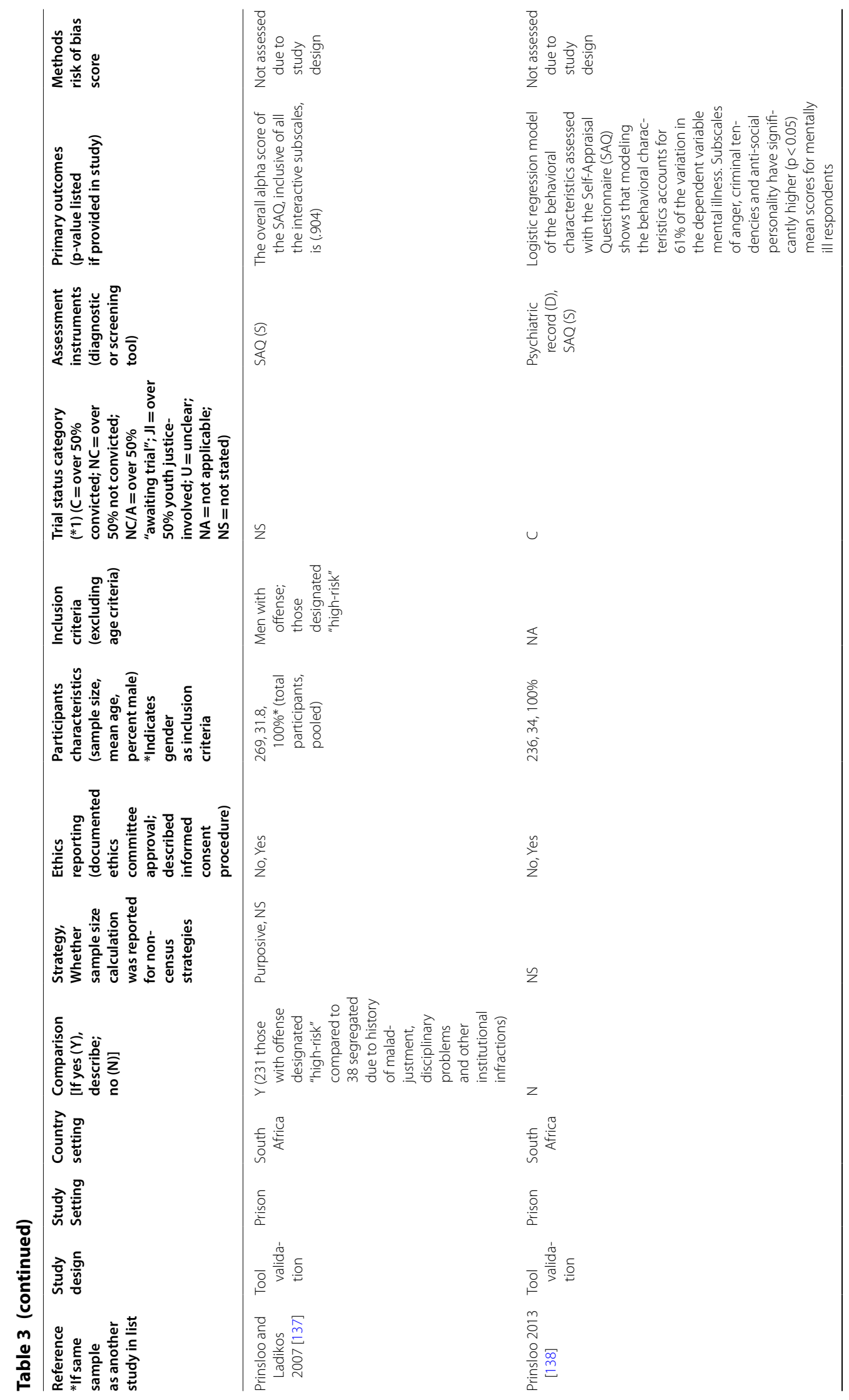




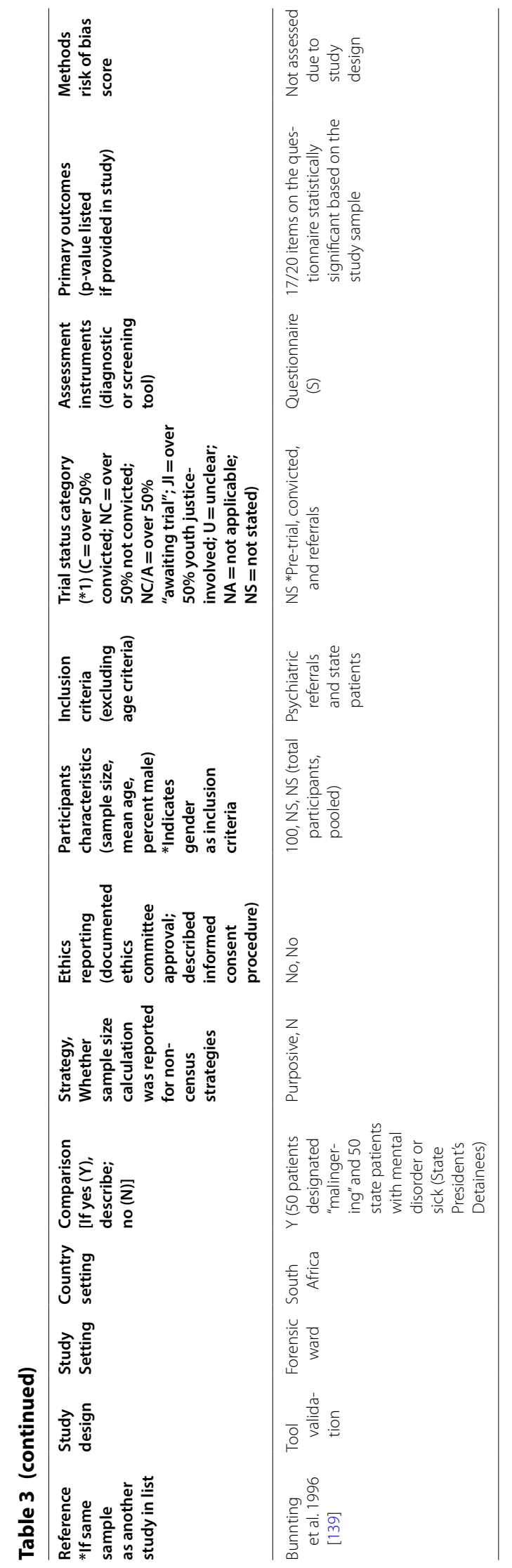


Table 4 Overall prevalence of mental disorders

\begin{tabular}{lllll}
\hline & Mental ill health & Mood disorder & Substance use & Psychotic disorders \\
\hline Pooled prevalence $(95 \% \mathrm{Cl})$ & $0.59(0.49-0.69)$ & $0.22(0.16-0.28)$ & $0.34(0.24-0.44)$ & $0.32(0.27-0.36)$ \\
${\text { Heterogeneity } \mathrm{chi}^{2}}^{2}$ & $2513.79(\mathrm{p}<0.001)$ & $3447.07(\mathrm{p}<0.001)$ & $1025.05(\mathrm{p}<0.001)$ & $3133.52(\mathrm{p}<0.001)$ \\
$\mathrm{I}^{2}$ & $99.09 \%$ & $99.19 \%$ & $98.24 \%$ & $99.14 \%$ \\
Number of studies & 24 & 29 & 19 & 28 \\
\hline
\end{tabular}

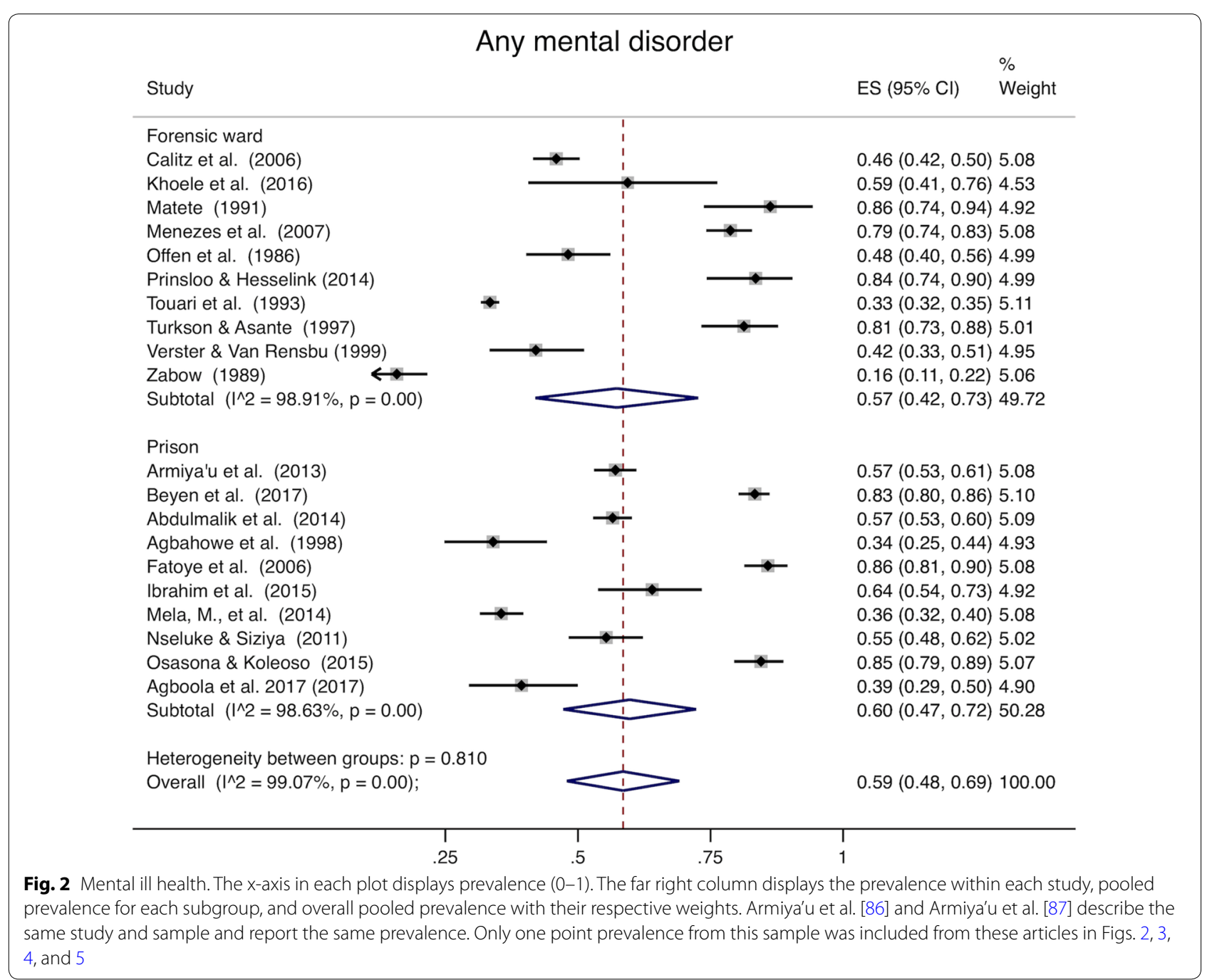

morbidity but not diagnose. Thirteen papers (23\%) reported outcomes obtained with screening tools alone, not diagnostic instruments. Reported factors associated with a psychiatric outcome and secondary outcomes are displayed in Additional file 1: Appendices S10 and S11.

\section{Prevalence studies}

Analysis of all studies combined (Table 4) was heterogeneous $\left(\mathrm{I}^{2}>98 \%\right.$ for all disease categories). When youth and adults were examined separately, pooled prevalence among adults was 59\% for mental ill health (95\% CI 48-69\%, Fig. 2), 22\% for mood disorders (95\% CI 16-28\%, Fig. 3), 33\% for psychotic disorders $(95 \%$ CI $28-37 \%$, Fig. 4), and $38 \%$ for substance use (95\% CI 26-50\%, Fig. 5). Among youth (Table 5), prevalence of mental ill health was $61 \%$ (95\% CI 17-100\%), mood disorder was $24 \%$ (95\% CI 14-25\%), and substance use was $22 \%$ (95\% CI 8-36\%). Heterogeneity analysis revealed 


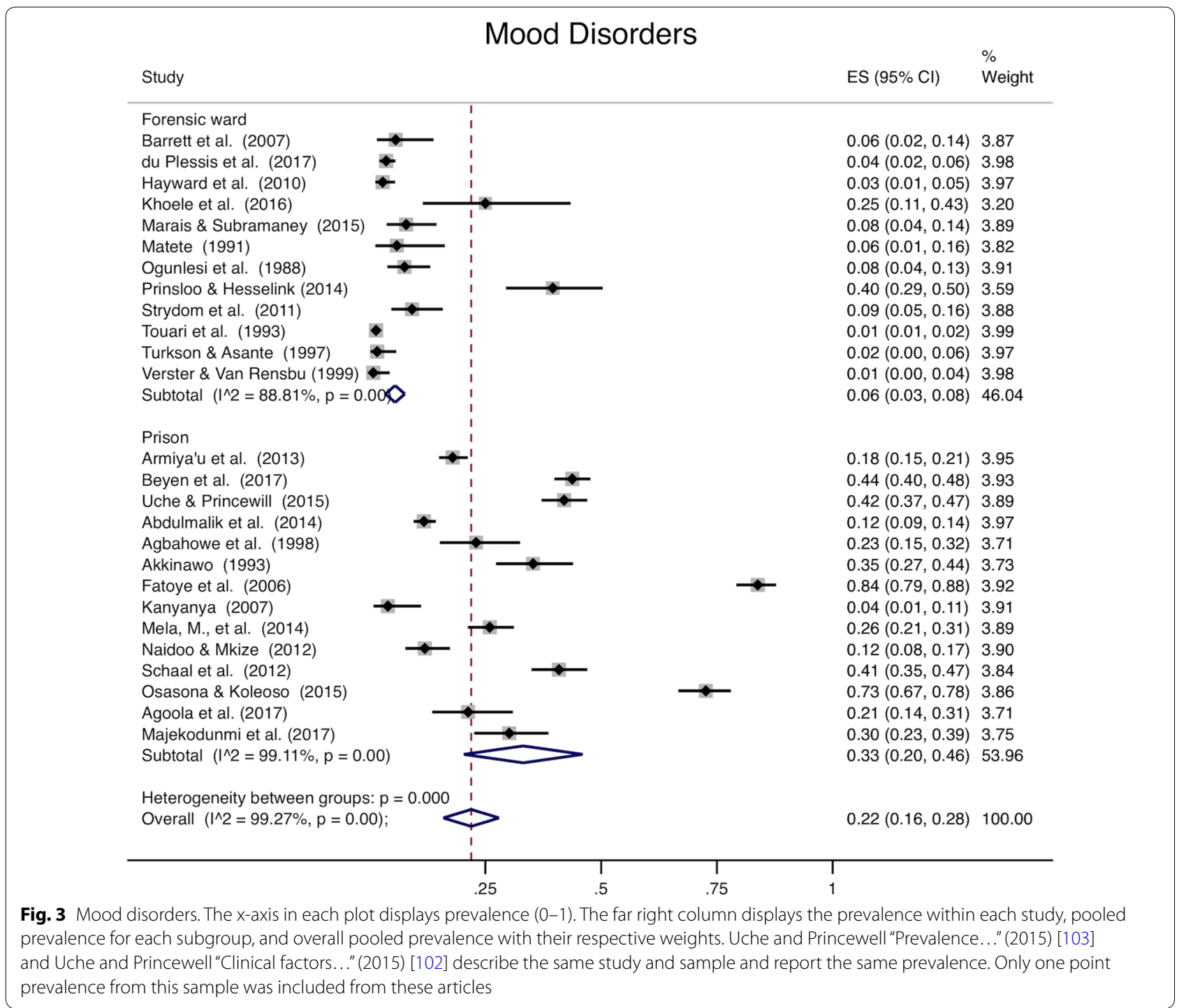

statistically significant heterogeneity for all disease categories and subgroup by institution (Figs. 2, 3, 4 and 5) $\left(\mathrm{I}^{2}>50 \%\right)$. The one exception was psychotic disorders in prisons, which because of their very low prevalence, were less heterogeneous $\left(\mathrm{I}^{2}=46.85 \%\right)$. The prevalence of psychotic disorders among inpatients in forensic wards was $44 \%$ (95\% CI 34-54\%), while in prisons the prevalence was $1 \%(95 \%$ CI $0-2 \%)$. Subgroup analysis by data collection method (clinical record or diagnostic/screening instrument) was not presented separately; all of the studies conducted in prisons use instruments to collect data, and all but one of those conducted in forensic institutions use clinical records. Thus, there is so much confounding that we cannot meaningfully separate the effects of data collection method from institution type. Robustness analysis (Additional file 1: Appendix S6) showed that the point prevalence estimates were similar regardless of how the subgrouping was done. Additionally, to examine if there was heterogeneity based on sampling technique, we conducted a sensitivity analysis including studies using census sampling only (the most homogenous sampling technique; randomization can vary substantially based on the method used to randomize, and the randomization method was not stated in most included studies) (Additional file 1: Appendix S7). Prevalence estimates of this subgrouping had as much heterogeneity as estimates from pooling different sampling methods (census, random, not stated) and were similar to overall estimates.

\section{Cross-sectional studies}

Nine cross-sectional studies did not collect psychiatric prevalence data, but investigated variables associated with mental health conditions, or justice or health system qualities. These studies examined associations between 


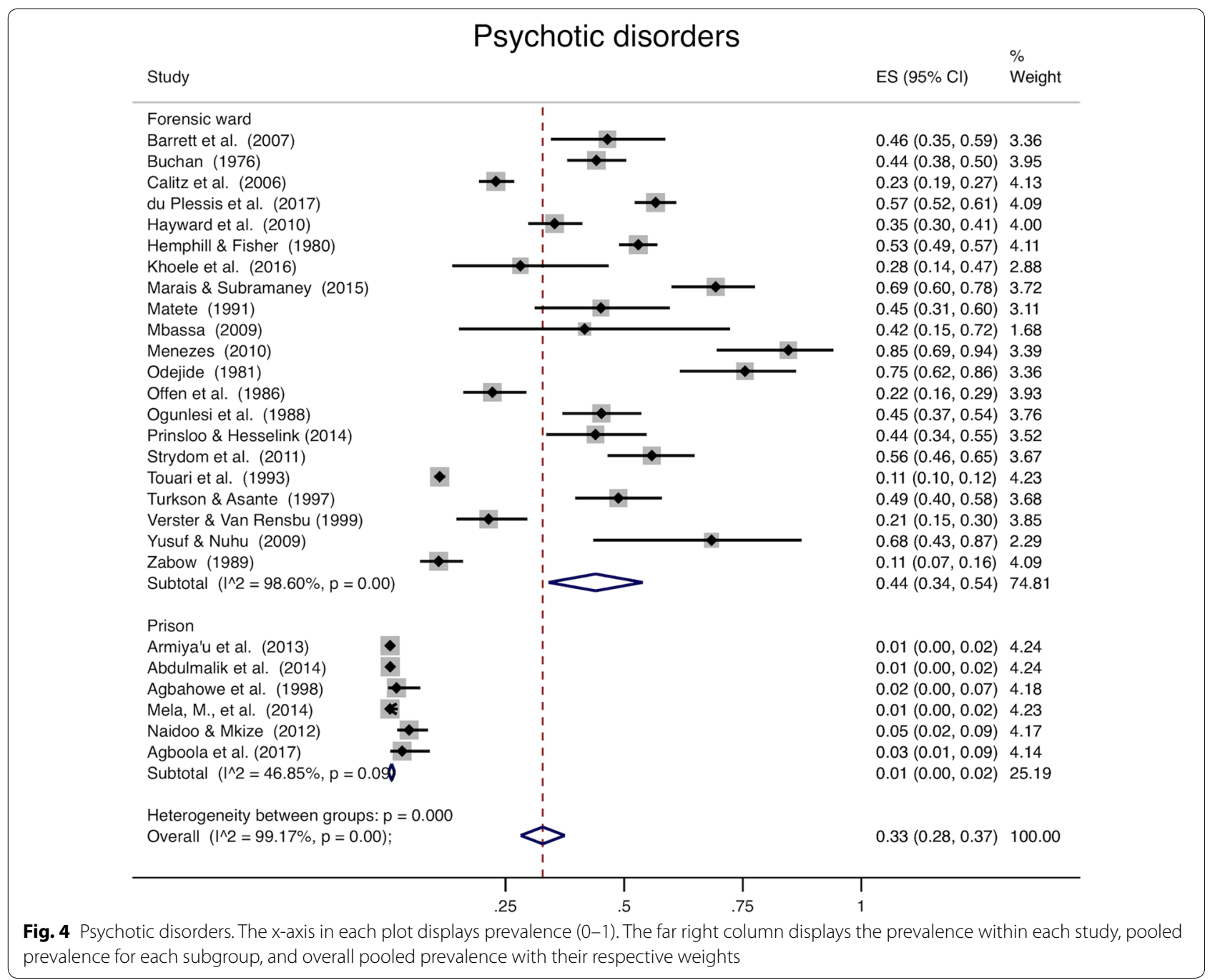

mental health-related variables (for instance, PTSD symptom severity [37]; substance misuse [38, 39]) and other factors (crimes committed [37]; prevalence of sexually transmitted infections [38]; emotional intelligence [40]). Alternatively, large and Nielssen found a positive correlation between per capita prison populations and per capita psychiatric hospital beds among LMICs and a combined pool of 158 countries, but no significant correlation among high-income countries [41].

\section{Interventions}

The intervention studies included two pre-post groupfocused cognitive behavioral interventions in prisons, one for cigarette smoking dependence and one for depression, both yielding significant improvements in the treatment group compared to the control group $(\mathrm{p}<0.001)[42,43]$. The single randomized control trial demonstrated significant decreases in schizophrenia symptoms following injections of two different neuroleptics in separate treatment arms (each neuroleptic treatment group resulted in a $\mathrm{p}<0.01$ decrease in the combined schizophrenia symptom score compared to the pre-injection score, and the Flupenthixol group showed a larger decrease than the Clopnethixol group $(\mathrm{p}$ $<0.01)$ ) [44]. Additional details of the intervention studies are listed in Additional file 1: Appendix S8.

\section{Qualitative studies}

The outcomes of the four qualitative studies included a shortage of medical personnel in prison mental health services in South Africa [45], poor prison conditions linked to mental health problems in a Zambian prison [46], psychiatric findings among women in prison for homicide [47], and gaps in awareness of the legal process and other legal characteristics of participants referred for psychiatric observation [48]. 


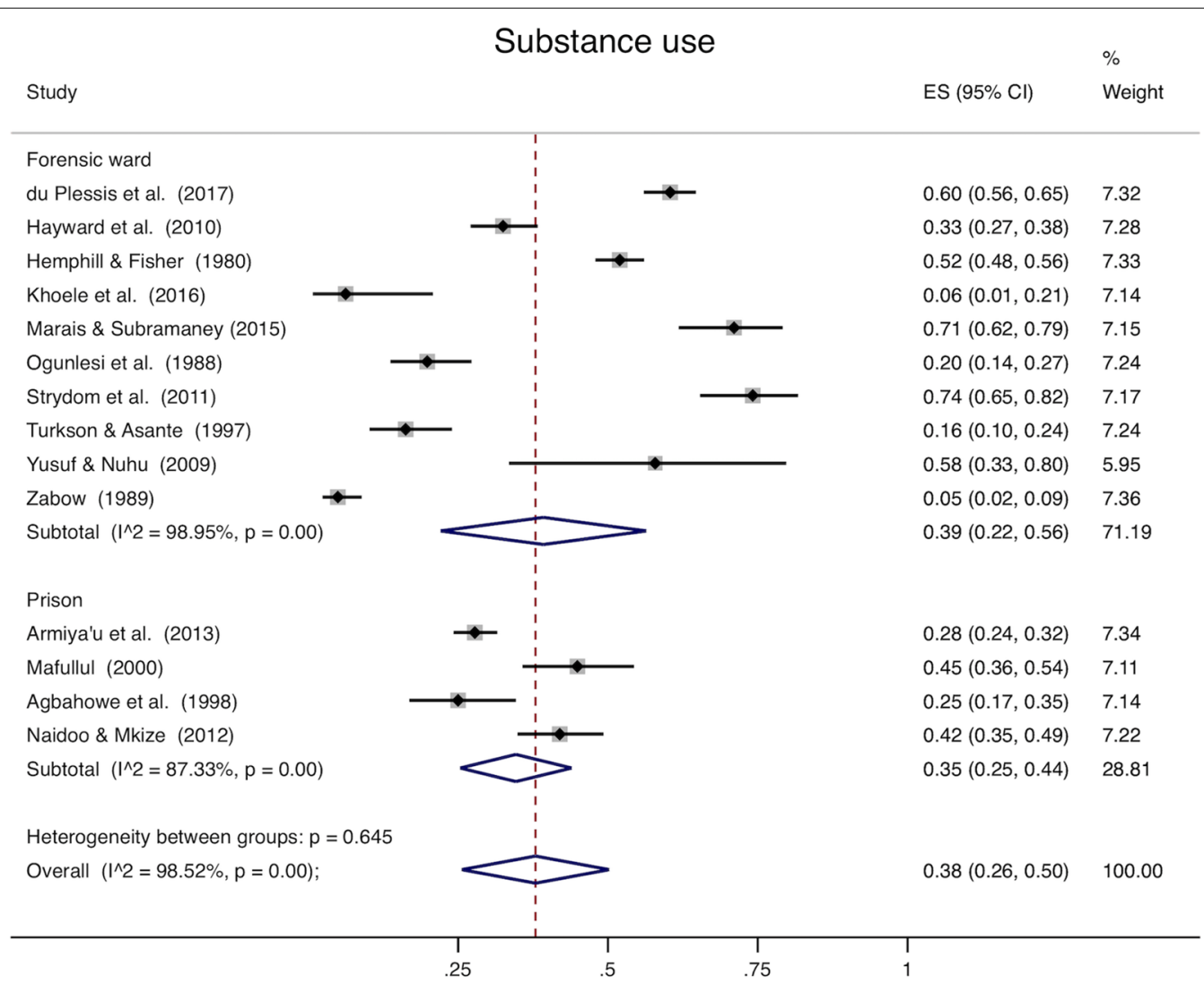

Fig. 5 Substance use. The $x$-axis in each plot displays prevalence (0-1). The far right column displays the prevalence within each study, pooled prevalence for each subgroup, and overall pooled prevalence with their respective weights

Table 5 Prevalence of mental disorders among youth

\begin{tabular}{llll}
\hline & Mental ill health & Mood disorder & Substance use \\
\hline Pooled prevalence $(95 \% \mathrm{Cl})$ & $0.61\left(0.17-1.00^{*}\right)$ & $0.24(0.14-0.35)$ & $0.22(0.08-0.36)$ \\
Heterogeneity $\mathrm{chi}^{2}$ & $422.59(\mathrm{p}<0.001)$ & $4.93(\mathrm{p}=0.08)$ & $95.75(\mathrm{p}<0.001)$ \\
$\mathrm{I}^{2}$ & $99.29 \%$ & $59.46 \%$ & $95.82 \%$ \\
Number of studies & 4 & 3 & 5 \\
\hline
\end{tabular}

Psychotic disorders were not included in this table, as only one youth study measured this outcome (Atilola 2012 "Prevalence and correlates..." [6])

*For clarity, this confidence interval is truncated at 1.00 as its upper bound

\section{Structured health systems reviews}

The two structured health systems reviews, both in Zimbabwe, each interviewed around 30 participants. One used an exploratory qualitative design, including interviews with people detained in the justice system, and proposed a model for transforming the medico-judicial system that involves multiple stages of mental health screening and diversion from the justice system [49]. The other used a structured needs assessment and interviews with policy makers, administrators, providers, and researchers to examine the national mental health system and found that many stakeholders called attention to the forensic mental health system although the researchers did not specifically ask about forensic mental health [22].

\section{Descriptions of conditions of detention, policy, law, and health systems}

We collected descriptions of mental health policy, laws, and systems if systematically collected and reported in any study type. Thirteen papers described conditions of detention in institutions, laws, or policy, including eight prevalence studies, three qualitative studies, and two structured health systems reviews. While it was not always clear whether the methodology used to report 
such outcomes was rigorous, we collected this data because of the scarcity of existing literature on this topic and report common findings thematically in Additional file 1: Appendix S9. The most common findings were insufficient human resources for health; lack of psychosocial services; lack of timely psychiatric assessment; and limited rehabilitation, recreational, vocational, or community re-integration services. Other common themes included insufficient physical resources, food, or psychiatric medicines; delays in trials, case-processing, or release; and lack of communication between medical and justice systems.

\section{Discussion}

To our knowledge, this is the first systematic review to investigate the mental health of PDJS in Africa exclusively. Results reflect that existing studies on this topic are predominantly prevalence studies that show a high pooled prevalence of mental illness, consistent with previous findings on mental illness in detained populations globally. Notably, many people detained within the justice system in non-prison locations, such as youth institutions or forensic hospitals, were detained with no charge $[6,7,50-55]$. The neglect of these populations in the literature is especially alarming in the context of pressures to deinstitutionalize mental healthcare in LMICs $[15,56-59]$.

A number of key populations were missing from our results. There were very few women included in the included studies, reflective of the small proportion of women found in prisons worldwide, particularly Africa, where only $3 \%$ of the total prison population are female, much lower than elsewhere [60]. Reasons for this may be that worldwide, likely due to distinct social roles, women commit fewer crimes [61] and that women are less likely to be convicted of crimes and sent to prison by courts [62]. The studies were also concentrated in a small number of African countries ( $73 \%$ of studies were in South Africa or Nigeria), and we found no studies in 36 of the 47 WHO-defined African countries. While more research is needed on translations of interventions across low-resource settings, we urgently need ground-work research in local contexts. Surprisingly, the general population detained in prisons was poorly represented in our sample, as prison studies were concentrated around people with particular psychiatric or forensic variables such as type of crime or trial status.

Our meta-analysis of prevalence studies revealed high pooled prevalence of mental disorders and substance use among PDJS in Africa, which underscores the urgency of addressing the mental health of detained people in Africa within the global mental health movement. However, the studies were heterogeneous. While we attempted to explore and explain the heterogeneity using subgroup analysis, nearly all subgroups were also heterogeneous. The most valid finding of the meta-analysis is that we have statistically shown that the studies were conducted on distinct populations. This is not surprising given that the populations are from across Africa and are detained in a variety of different types of institutions and under distinct penal policies. Accordingly, it is important to interpret the meta-analysis findings cautiously as the heterogeneity may limit the validity of providing a single point estimate for distinct populations. Notably, the level of heterogeneity we found was consistent with prior systematic reviews on the prevalence of mental disorders in prison settings [2]. All of this underscores the need for more research on mental health in prison settings and better standardization of the tools used to assess mental and substance use disorders.

While recognizing the heterogeneity, prevalence estimates are consistently higher than those estimated in a prior systematic review on mental illness in prison, which found a $3.6 \%$ prevalence of psychosis among men and $3.9 \%$ in women, and a $10.2 \%$ prevalence of major depression in men and $14.1 \%$ in women [2]. This prior review, however, included only one study from Africa, where resources for psychiatric care are particularly limited, and it did not include forensic wards. When we examined the prevalence of psychotic disorders in prisons alone (forensic psychiatry units excluded), the prevalence of psychotic disorders (1\%; 95\% CI 0.00-0.02) was much more similar to the prior review, but the prevalence of mood disorders (33\%; 95\% CI 0.20-0.46) remained higher. Although the prior review estimated the prevalence of major depression alone while this review includes bipolar disorder, almost all studies included in our estimate of mood disorders in prisons measured depression exclusively, and there was low prevalence of bipolar disorder. The prevalence of substance use disorder (35\%; 95\% CI $0.25-0.44$ ) was similarly high as observed in a prior international systematic review on substance use and dependence in prison, which found prevalence for alcohol use and dependence ranged from 18 to $30 \%$ among men and 10 to $24 \%$ among women, while prevalence of drug abuse and dependence varied from 10 to $48 \%$ in male prisoners and 30 to $60 \%$ in female prisoners [4]. There were too few women included in our study results to warrant meta-analysis by gender; however, the gender differences observed in prior research highlight the need for intentional data collection on gender in future work.

Subgroup analysis highlighted that there are differences in prevalence between institutions, with higher prevalence of mood disorders in prisons, higher prevalence of psychotic disorders in forensic institutions, and very low prevalence of psychotic disorders in prisons. This 
finding suggests that people with severe psychotic illnesses are being tracked out of prisons and into forensic units, which theoretically provide more psychiatric treatment. Interestingly, however, the prevalence of any sort of mental disorder in forensic units was less than $100 \%$, indicating that people without psychiatric diagnoses are being detained in these units in some settings. Additionally, there was relatively high prevalence of substance use across facilities, highlighting the importance of considering substance use when developing interventions in all settings of detention. The prevalence of any mental disorder in youth institutions had particularly high variability, perhaps because of the small number of studies and wide variety in reasons for youth detention. The findings of the meta-analysis, however, must be interpreted cautiously because the samples are highly heterogeneous, likely because these samples are drawn from different countries, institutions, and cultural contexts, and the outcomes are measured in a variety of ways with tools that may not be adequately validated in their settings. The heterogeneity is expected in a field with such paucity of data and is consistent with other meta-analyses of mental disorders in prisons [2].

Systematic reviews have the potential to include, and even elevate, ethically questionable studies due to the nature of an exhaustive search [31,63]. This may be particularly problematic for research involving vulnerable populations such as PDJS. We chose not to exclude any studies based on their ethical practices because excluding studies potentially detracts from knowledge that should eventually be used to help this vulnerable population. Thirty-five percent of studies failed to document ethics committee approval or informed consent procedures. Not all journals require ethics reporting, so our results do not necessarily indicate whether ethics procedures actually occurred, but raise concerns. The use of ethics assessment protocols in systematic reviews [31] and greater standardization for ethics reporting across journals could help ensure that all relevant ethical procedures are described.

The three effective interventions studies identified in the review may provide a starting point for future development of interventions and could be adapted for other settings. For instance, two interventions-one for depression [42], one for substance dependence [43] — used cognitive behavioral therapy approaches in group-focused sessions held twice a week. This group-focused, cognitive behavioral design could be adapted in future psychosocial interventions. The other intervention, a RCT for neuroleptics used to treat schizophrenia symptoms [44], highlights the strengths of pharmacological interventions for severe mental illness.

\section{Implications for health workers and policymakers}

First, we were surprised at how many studies included people who had not been convicted. Considerable work at all levels is needed to ensure that people with mental illness have rapid access to trial and are not detained without due process. Second, the high prevalence of mental disorders demonstrated across all settings of the justice system highlights the need for all staff members to receive mental health training. Likewise, those working in mental health should receive training on managing the needs of people who have been involved with the justice system. However, interventions must be coordinated and services designed to support individuals as they move across health, justice, and social systems. For instance, Munetz and Griffin propose the sequential intercept model for diversion of people with mental illness from the justice system in which various points of contact with the justice system become opportunities for connecting individuals to services; i.e. interceptions to prevent further justice involvement $[3,64]$. Third, the prevalence of substance use disorders was particularly high. Systematic reviews and RCTs from HICs highlight the importance of medication-assisted interventions, which combine psychological and pharmacological treatment, for substance use disorders in PDJS [3, 65, 66].

\section{Directions for future research}

First, the studies included have made it clear that there is high psychiatric prevalence among PDJS, but little development or testing of interventions to address the large mental health need. Contextual research on each system of detention and subpopulation, including youth, and older participants, trial or charge status, gender, and diagnosis, is essential to inform such interventions. In addition to providing mental health services within institutions of detention, the high prevalence of mental illness compared to the general populations suggests a need for research on supportive diversions of individuals with mental illness from the justice system to best connect individuals with care. Second, we have focused on the academic literature, but there should also be systematic and comprehensive data collection and analysis of institutional and governmental documents surrounding the mental health of PDIS in Africa. Third, there is need for longitudinal studies that follow participants to community re-entry, especially investigating transitions to community health care and long-term health and recidivism outcomes $[67,68]$. Fourth, only five studies in this review collected qualitative data from PDJS themselves. Studies must do more to include service user voices. Fifth, prisons, youth institutions, and forensic psychiatry settings must be investigated both discretely and as facilities that feed into each other: each settings' population 
has specific needs, but there may be revolving door effect for forensic psychiatric units and prisons, as described in Zimbabwe [49]. Sixth, economics research was not present in our results, but is needed to measure the societal costs of lack of treatment, the cost-effectiveness of treatments, and the potential cost-savings provided by interventions and diversions.

\section{Limitations}

First, a major limitation for this review was the heterogeneity of studies and overall paucity of high quality literature. We chose broader definitions of detention and mental illness to provide a comprehensive perspective on this understudied area and better define the state of the field in Africa. However, because results were more variable than expected, we were unable to analyze specific aspects or subpopulations (interventions, forensic units, youth institutions, policy) in as much depth as we would have liked. We aim to delve deeper in the future, producing discrete publications on forensic hospitals, youth institutions, conditions of detention, but reported all results of this paper collectively in accordance with PRISMA guidelines [23], and to avoid legal, ethical, and methodological issues that arise from post hoc changes to the protocol and attempts to publish slices or versions of data that have been published previously [69]. This heterogeneity in included studies makes it challenging to point to singular conclusions from the data. However, the heterogeneity of study settings is a strength since exhaustive inclusion allows us to speak to the state of the issue in Africa at large and gives readers a more comprehensive overview of key areas for future work. This approach calls attention to the systems-wide nature of detention of people with mental illness, whereas prior reviews have excluded non-prison populations with high psychiatric prevalence that are similarly detained by the state. Second, because we added the meta-analysis post hoc, it was not included in our original protocol. We did not select studies with the aim of having a homogenous set of outcomes or study designs to facilitate meta-analysis. Additionally, the disease categories we used to group analysis were generated inductively based on the included studies. They were broad and likely introduced additional heterogeneity into the groups. However, we believe that metaanalysis provides one additional way for readers to access and understand our data and triangulates the results of the narrative review, which were consistent with the meta-analysis findings. Third, we discovered eight studies during the backward search: a signal that perhaps the original search strategy was imperfect. Many of these studies were from small journals that were not indexed in the major databases that we had originally chosen to search. While we could have iteratively changed the search strategy to broaden the list of databases we searched, we chose to search only large databases of established quality and to adhere to our study protocol. Finally, a unifying theme throughout our review and in evaluating our limitations has been a data quality issue, as many studies had high risk of bias or reported so little about their methods that it was difficult to assess risk of bias.

\section{Conclusion}

This review has identified key areas that require further research, and demonstrated need for more standardized methods and ethics reporting. It has confirmed the high prevalence of mental illness among PDJS in Africa, but revealed an absence of setting diversity or diversity of study types, and revealed key populations underresearched or missing from the literature. Though the need for bio-medically focused interventions is clear from this high psychiatric prevalence, we look forward to a future in which prevention approaches and social interventions are prioritized. Social factors of stress, poverty, and discrimination may disproportionately affect people that become detained and contribute to poor mental health. Future mental health research must take on a systems-wide perspective involving both the health and justice sectors, and investigate both clinical and contextual social variables. This approach will guide interventions for coordinated service development, and better align policy with the aim of the Sustainable Development Goals' to leave no one behind in achieving equitable, universal health coverage $[70,71]$.

\section{Additional file}

Additional file 1. Appendices S1-S11.

\section{Authors' contributions}

$\mathrm{HJ}, \mathrm{KK}$, and GT conceived the project. HJ drafted the protocol with input from GF. HJ designed and conducted the literature search. AL, HK, and MC screened studies for inclusion, completed the methodology review, and extracted data, with guidance from $\mathrm{HJ}$. AL drafted the manuscript with input from $\mathrm{HK}$ and $\mathrm{MC}$. DM, GF, and GT provided supervision and ongoing feedback during the study. All authors reviewed and edited subsequent versions of the manuscript. HJ conducted meta-analysis. All authors read and approved the final manuscript.

\section{Author details}

${ }^{1}$ Harvard College, 28 Fernald Drive, Cambridge, MA 02138, USA. ${ }^{2}$ Institute of Psychology, Psychiatry, and Neuroscience, King's College London, 16 De Crespigny Park, Camberwell, London SE5 8AB, UK. ${ }^{3}$ Kushinga, 8 Collina Close, Borrowdale, Harare, Zimbabwe. ${ }^{4}$ Brigham and Women's Hospital, 75 Francis Street, Boston, MA 02115, USA. ${ }^{5}$ Department of Psychiatry, University of Zimbabwe, 630 Churchill Avenue, Harare, Zimbabwe. ${ }^{6}$ The Ulster Hospital, Upper Newtownards Road, Dundonald, Belfast BT16 1RH, UK. ${ }^{7}$ Department of Psychiatry, Massachusetts General Hospital, 55 Fruit Street, Boston, MA 02114, USA. ${ }^{8}$ Centre for Global Mental Health, Institute of Psychology, Psychiatry, and Neuroscience, King's College London, London, UK. ${ }^{9}$ Department of Medicine, University of Washington, 1959 NE Pacific Street, Seattle, WA 98195, USA. 


\begin{abstract}
Acknowledgements
We thank our colleagues at Kushinga, reference librarian Paul Bain, and John Choe, MD for their guidance on this project. We thank the staff members of Harvard Interlibrary Loan for their work in locating the texts of dozens of papers not available in the database, especially full-time library staff members Addie Owens, Anne-Marie Taylor, Desiree Goodwin, Katie DeFord, and Zemtawork Tesfaye among others. Author GT is supported by the National Institute for Health Research (NIHR) Collaboration for Leadership in Applied Health Research and Care South London at King's College London NHS Foundation Trust. The views expressed are those of the author(s) and not necessarily those of the NHS, the NIHR or the Department of Health. GT also receives support from the National Institute of Mental Health of the National Institutes of Health under award number R01MH100470 (Cobalt study). GT is also supported by the UK Medical Research Council in relation the Emilia (MR/ S001255/1) and Indigo Partnership (MR/R023697/1) awards.
\end{abstract}

\section{Competing interests}

The authors declare that they have no competing interests.

\section{Availability of data and materials}

All data supporting the conclusions of this article are extracted from primary literature and included in Tables 2 and 3 and in the Additional file 1: Appendix.

\section{Consent for publication}

Not applicable.

\section{Ethics approval and consent to participate}

Not applicable; all data presented is from previously published literature.

\section{Funding}

We received no funding for this research specifically. However, author GT acknowledges financial support from the Department of Health via the National Institute for Health Research (NIHR) Biomedical Research Centre and Dementia Unit awarded to South London and Maudsley NHS Foundation Trust in partnership with King's College London and King's College Hospital NHS Foundation Trust. GT is supported by the European Union Seventh Framework Programme (FP7/2007-2013) Emerald project. GT also receives support from the National Institute of Mental Health of the National Institutes of Health under award number R01MH100470 (Cobalt study). GT is also supported by the UK Medical Research Council in relation the Emilia and Indigo Partnership awards.

\section{Publisher's Note}

Springer Nature remains neutral with regard to jurisdictional claims in published maps and institutional affiliations.

\section{Received: 27 November 2018 Accepted: 15 March 2019}

Published online: 06 May 2019

\section{References}

1. Vigo D, Thornicroft G, Atun R. Estimating the true global burden of mental illness. Lancet Psychiatry. 2016;3(2):171-8.

2. Fazel S, Seewald K. Severe mental illness in 33,588 prisoners worldwide: systematic review and meta-regression analysis. Br J Psychiatry. 2012;200(5):364-73

3. Fazel S, Hayes AJ, Bartellas K, Clerici M, Trestman R. Mental health of prisoners: prevalence, adverse outcomes, and interventions. Lancet Psychiatry. 2016;3(9):871-81.

4. Fazel $S$, Bains $P$, Doll $H$. Substance abuse and dependence in prisoners: a systematic review. Addiction. 2006;101(2):181-91.

5. Fazel S, Danesh J. Serious mental disorder in 23000 prisoners: a systematic review of 62 surveys. Lancet. 2002;359(9306):545-50.

6. Atilola O. Prevalence and correlates of psychiatric disorders among residents of a juvenile Remand Home in Nigeria: implications for mental health service planning. Niger J Med. 2012;21(4):416-26.

7. Atilola O, Omigbodun O, Bella-Awusah T. Post-traumatic stress symptoms among juvenile offenders in Nigeria: implications for holistic service provisioning in juvenile justice administration. J Health Care Poor Underserved. 2014;25(3):991-1004.

8. El-Gilany A, Khater M, Gomaa Z, Hussein E, Hamdy I. Psychiatric disorders among prisoners: a national study in Egypt. East Asian Arch Psychiatry. 2016;26(1):30-8.

9. Sirdifield C. A systematic review of research on the epidemiology of mental health disorders in prison populations: a summary of findings. J Forensic Psychiatry Psychol. 2009;20(S1):S78-101.

10. WHO. Mental health atlas 2017. Geneva: WHO; 2018.

11. Walmsley R. World prison population list. 11th ed. London: Institute for Criminal Policy Research, Birkbeck University of London; 2016.

12. WHO. Mental health atlas 2014. Geneva: WHO; 2015.

13. Winkler P, Krupchanka D, Roberts T, Kondratova L, Machu V, Hoschl C, Sartorius N, Van Voren R, Aizberg O, Bitter I, et al. A blind spot on the global mental health map: a scoping review of 25 years' development of mental health care for people with severe mental illnesses in central and eastern Europe. Lancet Psychiatry. 2017;4(8):634-42.

14. Petersen I, Bhana A, Campbell-Hall V, Mjadu S, Lund C, Kleintjies S, Hosegood V, Flisher AJ. Planning for district mental health services in South Africa: a situational analysis of a rural district site. Health Policy Plan. 2009;24(2):140-50.

15. WHO. The World Health Report 2001: mental health: new understanding, new hope. Geneva:WHO; 2001. p. 2001.

16. Onah ME. The patient-to-prisoner pipeline: the IMD exclusion's adverse impact on mass incarceration in United States. Am J Law Med. 2018;44(1):119-44.

17. Lamb HR, Weinberger LE. The shift of psychiatric inpatient care from hospitals to jails and prisons. J Am Acad Psychiatry Law. 2005;33(4):529-34.

18. Winkler P, Barrett B, McCrone P, Csemy L, Janouskova M, Hoschl C. Deinstitutionalised patients, homelessness and imprisonment: systematic review. Br J Psychiatry. 2016;208(5):421-8.

19. Jack HE, Fricchione G, Chibanda D, Thornicroft G, Machando D, Kidia K. Mental health of incarcerated people: a global call to action. Lancet Psychiatry. 2018;5(5):391-2.

20. Yoon IA, Slade K, Fazel S. Outcomes of psychological therapies for prisoners with mental health problems: a systematic review and metaanalysis. J Consult Clin Psychol. 2017;85(8):783-802.

21. Goomany A, Dickinson T. The influence of prison climate on the mental health of adult prisoners: a literature review. J Psychiatr Ment Health Nurs. 2015;22(6):413-22.

22. Kidia K, Machando D, Mangezi W, Hendler R, Crooks M, Abas M, Chibanda D, Thornicroft G, Semrau M, Jack H. Mental health in Zimbabwe: a health systems analysis. Lancet Psychiatry. 2017;4(11):876-86.

23. Moher D, Liberati A, Tetzlaff J, Altman DG. Preferred reporting items for systematic reviews and meta-analyses: the PRISMA statement. J Clin Epidemiol. 2009;62(10):1006-12.

24. Stroup DF, Berlin JA, Morton SC, Olkin I, Williamson GD, Rennie D, Moher D, Becker BJ, Sipe TA, Thacker SB. Meta-analysis of observational studies in epidemiology: a proposal for reporting. J Am Med Assoc. 2000;283(15):2008-12.

25. Countries, WHO Regional Office for Africa. http://www.afro.who.int/ countries. Accessed 7 Jun 2018

26. Thomas H. Quality assessment tool for quantitative studies. Toronto: Effective Public Health Practice Project McMaster University; 2003.

27. Higgins JPT. The Cochrane Collaboration's tool for assessing risk of bias in randomised trials. BMJ. 2011;343(7829):889-93.

28. CASP Qualitative Research Checklist. In: Critical Appraisal Skills Programme; 2018.

29. Aromataris EFR, Godfrey C, Holly C, Kahlil H, Tungpunkom P. Summarizing systematic reviews: methodological development, conduct and reporting of an Umbrella review approach. Int J Evid Based Healthc. 2015;13(3):132-40.

30. Juni $P$, Witschi $A$, Bloch $R$, Egger $M$. The hazards of scoring the quality of clinical trials for meta-analysis. J Am Med Assoc. 1999;282(11):1054-60.

31. Weingarten MA, Paul M, Leibovici L. Assessing ethics of trials in systematic reviews. BMJ. 2004;328(7446):1013-4.

32. Nyaga VN, Arbyn M, Aerts M. Metaprop: a Stata command to perform meta-analysis of binomial data. Arch Public Health. 2014;72(1):39.

33. Goldberg DP, Hillier VF. A scaled version of the General Health Questionnaire. Psychol Med. 1979;9(1):139-45. 
34. Higgins JP, Thompson SG, Deeks JJ, Altman DG. Measuring inconsistency in meta-analyses. BMJ. 2003;327(7414):557-60.

35. Petitti D. Meta-analysis, decision analysis, and cost-effectiveness analysis. Methods for quantitative synthesis in medicine, vol. 24., Monographs in epidemiology and biostatisticsNew York: Oxford University Press; 1994.

36. StataCorp. Stata Statistical Software: Release 15. College Station: StataCorp LLC; 2017.

37. Weierstall R, Schaal S, Schalinski I, Dusingizemungu JP, Elbert T. The thrill of being violent as an antidote to posttraumatic stress disorder in Rwandese genocide perpetrators. Eur J Psychotraumatol. 2011. https:// doi.org/10.3402/ejpt.v2i0.6345.

38. Stephens T, Braithwaite RL, Reddy PS, Sifunda S, Bhengu S. Lifetime occurrence of sexually transmitted infection (STI) and substance use risk among prerelease South African prison inmates. Int Q Community Health Educ. 2006;26(4):355-63.

39. Ononye F, Morakinyo O. Drug abuse, psychopathology and juvenile delinquency in south-western Nigeria. J Forensic Psychiatry. 1994;5(3):5257-538.

40. Bgo A. Correlates of depression among prison inmates in South-Western Nigeria. Niger J Appl Behav Sci. 2013;1:43-55.

41. Large MM, Nielssen O. The Penrose hypothesis in 2004: patient and prisoner numbers are positively correlated in low-and-middle income countries but are unrelated in high-income countries. Psychol Psychother. 2009:82(Pt 1):113-9.

42. Eseadi C, Obidoa MA, Ogbuabor SE, Ikechukwu-llomuanya AB. Effects of group-focused cognitive-behavioral coaching program on depressive symptoms in a sample of inmates in a Nigerian prison. Int J Offender Ther Comp Criminol. 2017;62(6):1589-1602. https://doi. org/10.1177/0306624X16687046.

43. Onyechi KC, Eseadi C, Umoke PC, Ikechukwu-Ilomuanya AB, Otu MS, Obidoa JC, Agu FU, Nwaubani OO, Utoh-Ofong AN, Ncheke CD, et al. Effects of a group-focused cognitive behavioral health education program on cigarette smoking in a sample of Nigerian prisoners. Medicine. 2017:96(1):e5158.

44. Martyns-Yellowe IS. The decanoates of flupenthixol and clopenthixol in the treatment of chronic schizophrenic in-patients. Implications for community psychiatry. West Afr J Med. 1993;12(2):110-3.

45. Gaum G, Hoffman S, Venter JH. Factors that influence adult recidivism: an exploratory study in Pollsmoor prison. S Afr J Psychol. 2006;36(2):407-24.

46. Topp SM, Moonga CN, Luo N, Kaingu M, Chileshe C, Magwende G, Heymann SJ, Henostroza G. Exploring the drivers of health and healthcare access in Zambian prisons: a health systems approach. Health Policy Plan. 2016;31(9):1250-61.

47. Pretorius G, Bester M. Abused women in South Africa who kill their intimate male partners: a psychological examination. J Psychol Africa. 2009;19(3):371-8

48. Kaliski SZ, Boreherds M, Williams F. Defendants are clueless-the 30-day psychiatric observation. S Afr Med J. 1997;87(10):1351-5.

49. Dube-Mawerewere V. A medico-judicial framework for the rehabilitation of forensic psychiatric patients in Zimbabwe. J Forensic Pract. 2015;17(2):134-48.

50. Atilola O, Ola B, Abiri G. Service and Policy implication of substance use disorders among adolescents in juvenile correctional facilities in Lagos, Nigeria. Glob Ment Health. 2016;3:e30.

51. Bella TT, Atilola O, Omigbodun OO. Children within the juvenile justice system in Nigeria: psychopathology and psychosocial needs. Ann Ib Postgrad Med. 2010;8(1):34-9.

52. Barrett SP, du Plooy J, du Toit J, Wilmans S, Calitz FJW, Joubert G. Profile of mentally ill offenders referred to the free state psychiatric complex. $S$ Afr J Psychiatry. 2007;13(2):56-8.

53. Marais B, Subramaney U. Forensic state patients at sterkfontein hospital: a 3-year follow-up study. Afr J Psychiatry. 2015;21(3):86-92.

54. Strydom N, Pienaar C, Dreyer A, van der Merwe L, van Rensburg BJ, Calitz FJW, van der Merwe LM, Joubert G. Profile of forensic psychiatric inpatients referred to the free state psychiatric complex, 2004-2008. S Afr J Psychiatry. 2011;17(2):40-3.

55. Turkson SN, Asante K. Psychiatric disorders among offender patients in the Accra Psychiatric Hospital. West Afr J Med. 1997;16(2):88-92.
56. Freeman MC Global lessons for deinstitutionalisation from the illfated transfer of mental health-care users in Gauteng, South Africa. Lancet Psychiatry. 2018;5(9):765-8. https://doi.org/10.1016/S2215 -0366(18)30211-6.

57. Patel V, Belkin GS, Chockalingam A, Cooper J, Saxena S, Unutzer J. Grand challenges: integrating mental health services into priority health care platforms. PLoS Med. 2013;10(5):e1001448.

58. Ngo VK, Rubinstein A, Ganju V, Kanellis P, Loza N, Rabadan-Diehl C, Daar AS. Grand challenges: integrating mental health care into the noncommunicable disease agenda. PLoS Med. 2013;10(5):e1001443.

59. WHO. Innovation in deinstitutionalization: a WHO expert survey. Geneva:WHO; 2014.

60. Walmsley R. World female imprisonment list: women and girls in penal institutions, including pre-trial detainees/remand prisoners. 4th edn. World Prison Brief; 2017.

61. Becker S, McCorkel JA. The gender of criminal opportunity: the impact of male co-offenders on women's crime. Feminist Criminol. 2011:6(2):79-110

62. Doerner JK, Demuth S. Gender and sentencing in the federal courts: are women treated more leniently? Crim Justice Policy Rev. 2014;25(2):242-69.

63. Vergnes JN, Marchal-Sixou C, Nabet C, Maret D, Hamel O. Ethics in systematic reviews. J Med Ethics. 2010;36(12):771-4.

64. Munetz MR, Griffin PA. Use of the sequential intercept model as an approach to decriminalization of people with serious mental illness. Psychiatr Serv. 2006;57(4):544-9.

65. Koehler JAHD, Akoensi TD, de Ribera OS, Loesel F. A systematic review and meta-analysis on the effects of European drug treatment programmes on reoffending. Psychol Crime Law. 2014;20:584-602.

66. Perry AE, Neilson M, Martyn-St James M, Glanville JM, Woodhouse R, Godfrey C, Hewitt C. Pharmacological interventions for drug-using offenders. Cochrane Database Syst Rev. 2015;6:CD010862.

67. Hopkin G, Evans-Lacko S, Forrester A, Shaw J, Thornicroft G. Interventions at the transition from prison to the community for prisoners with mental illness: a systematic review. Adm Policy Ment Health. 2018:45(4):623-34.

68. Stewart LA, Farrell-MacDonald S, Feeley S. The impact of a community mental health initiative on outcomes for offenders with a serious mental disorder. Crim Behav Ment Health. 2017;27(4):371-84.

69. Recommendations: Overlapping Publications. http://www.icmje.org/ recommendations/browse/publishing-and-editorial-issues/overlappin g-publications.html. Accessed 18 Jan 2019.

70. UN. Global indicator framework for the sustainable development goals and targets of the 2030 agenda for sustainable development. UN: United Nations Statistical Commission; 2018.

71. Patel V, Saxena S, Lund C, Thornicroft G, Baingana F, Bolton P, Chisholm D, Collins PY, Cooper JL, Eaton J, et al. The Lancet Commission on global mental health and sustainable development. Lancet. 2018:392(10157):1553-98. https://doi.org/10.1016/S0140 $-6736(18) 31612-X$.

72. WHO. Mental health action plan 2013-2020. Geneva: WHO; 2013.

73. WHO. Time to deliver, report of the WHO independent high-level commission on noncommunicable diseases. Geneva:WHO; 2018.

74. WHO. World Health Organization assessment instrument for mental health systems. Geneva:WHO; 2005.

75. WHO. Mental health and development: targeting people with mental health conditions as a vulnerable group. Geneva: WHO; 2010.

76. WHO. Checklist for evaluating a mental health policy. Geneva: WHO Mental Health Policy and Service Guidance Package; 2005.

77. UN. Report of the United Nations High Commissioner for human rights: mental health and human rights. UN General Assembly; 2017.

78. UN. Technical guidance for prison planning: technical and operational considerations based on the Nelson Mandela rules. Copenhagen: United Nations Office for Project Services; 2016.

79. UN. Handbook on prisoners with special needs., Criminal justice handbook seriesNew York: UNODC; 2009.

80. UN. United Nations expert group meeting on mental well-being, disability and disaster risk reduction. Tokyo: United Nations University; 2014.

81. UN. United Nations standard minimum rules for the treatment of prisoners (the Nelson Mandela Rules). 2015. 
82. Abdulmalik JO, Adedokun BO, Baiyewu OO. Prevalence and correlates of mental health problems among awaiting trial inmates in a prison facility in Ibadan, Nigeria. Afr J Med Med Sci. 2014;43(Suppl 1):193-9.

83. Agbahowe SA, Ohaeri JU, Ogunlesi AO, Osahon R. Prevalence of psychiatric morbidity among convicted inmates in a Nigerian prison community. East Afr Med J. 1998;75(1):19-26.

84. Agboola A, Babalola E, Udofia O. Psychopathology among offenders in a Nigeria prison. Int J Clin Psychiatry. 2017;5(1):10-5.

85. Akinnawo EO. Prevalence of psychological symptoms in a Nigerian prison. Psychopathol De Aticaine XXV. 1993;1:93-104.

86. Armiya'u AY, Obembe A, Audu MD, Afolaranmi TO. Prevalence of psychiatric morbidity among inmates in Jos maximum security prison. Open J Psychiatry. 2013;3:12-7.

87. Armiya'u AY, Audu MD, Obembe A, Adole O, Umar MU. A study of psychiatry morbidity and co-morbid physical illness among convicted and awaiting trial inmates in Jos prison. J Forensic Legal Med. 2013;20(8):1048-51

88. Beyen TK, Dadi AF, Dachew BA, Muluneh NY, Bisetegn TA. More than eight in every nineteen inmates were living with depression at prisons of Northwest Amhara Regional State, Ethiopia, a cross sectional study design. BMC Psychiatry. 2017;17(1):31.

89. Dachew BA, Fekadu A, Kisi T, Yigzaw N, Bisetegn TA. Psychological distress and associated factors among prisoners in North West Ethiopia: cross-sectional study. Int J Ment Health Syst. 2015:9:39.

90. Dadi AF, Dachew BA, Kisi T, Yigzaw N, Azale T. Anxiety and associated factors among prisoners in North West of Amhara Regional State, Ethiopia. BMC Psychiatry. 2016;16:83

91. Fatoye FO, Fatoye GK, Oyebanji AO, Ogunro AS. Psychological characteristics as correlates of emotional burden in incarcerated offenders in Nigeria. East Afr Med J. 2006;83(10):545-52.

92. Ibrahim A, Esena RK, Aikins M, O'Keefe AM, McKay MM. Assessment of mental distress among prison inmates in Ghana's correctional system: a cross-sectional study using the Kessler Psychological Distress Scale. Int J Ment Health Syst. 2015;9:17.

93. Kanyanya IM, Othieno CJ, Ndetei DM. Psychiatric morbidity among convicted male sex offenders at Kamiti prison, Kenya. East Afr Med J. 2007:84(4):151-5.

94. Mafullul YM. Homicide in northern Nigeria: a clinico-forensic survey. Am J Forensic Psychiatry. 2000;21(3):69-88

95. Mafullul YM, Ogunlesi OA, Sijuwola OA. Psychiatric aspects of criminal homicide in Nigeria. East Afr Med J. 2001;78(1):35-9.

96. Majekodunmi OE, Obadeji A, Oluwole LO, Oyelami RO. Depression in prison population: demographic and clinical predictors. J Forensic Sci Med. 2017;3(3):122-7.

97. Mela M, Audu M, Tesfaye M, Gurmu S. A developing world perspective on homicide and personality disorder. Med Sci Law. 2014:54(3):132-8.

98. Naidoo S, Mkize DL. Prevalence of mental disorders in a prison population in Durban, South Africa. Afr J Psychiatry. 2012;15(1):30-5.

99. Nseluke MSS. Prevalence and socio-demographic correlates for mental illness among inmates at Lusaka Central Prison. Zamb Med J. 2011:38:10-20.

100. Osasona SO, Koleoso ON. Prevalence and correlates of depression and anxiety disorder in a sample of inmates in a Nigerian prison. Int J Psychiatry Med. 2015;50(2):203-18.

101. Schaal S, Weierstall R, Dusingizemungu JP, Elbert T. Mental health 15 years after the killings in Rwanda: imprisoned perpetrators of the genocide against the Tutsi versus a community sample of survivors. J Trauma Stress. 2012;25(4):446-53.

102. Uche N, Princewill S. Clinical factors as predictors of depression in a Nigerian prison population. J Psychiatry. 2015. https://doi. org/10.4172/2378-5756.1000345.

103. Uche N, Princewill S. Prevalence of depression in Port Harcourt Prison. J Psychiatry. 2015. https://doi.org/10.4172/2378-5756.1000340.

104. Buchan T. Some problems in the hospital management of criminal mental patients. S Afr Med J. 1976;50(32):1252-6.

105. Calitz FJW, van Rensburg PHJJ, Fourie C, Liebenberg E, van den Berg C, Joubert G. Psychiatric evaluation of offenders referred to the Free State Psychiatric Complex according to sextions 77 and/or 78 of the Criminal Procedures Act. S Afr J Psychiatry. 2006;12(3):47-51.

106. du Plessis ED, du Plessis HJ, Nel HC, Oosthuizen I, van der Merwe S, Zwiegers S, Joubert G. Accountable or not accountable: a profile comparison of alleged offenders referred to the free state psychiatric complex forensic observation ward in Bloemfontein from 2009 to 2012. S Afr J Psychiatry. 2017;23:a1054. https://doi.org/10.4102/sajps ychiatry.v23i0.1054.

107. Hayward D, White T, Kauye F. Review of the forensic psychiatry service at Zomba Mental Hospital and a comparison of forensic psychiatry services in Malawi and Scotland. Med Sci Law. 2010;50(2):67-71.

108. Hemphill RE, Fisher W. Drugs, alcohol and violence in 604 male offenders referred for inpatient psychiatric assessment. S Afr Med J. 1980;57(7):243-7

109. Khoele KB, de Wet PH, Pretorius HW, Sommerville J. Case series of females charged with murder or attempted murder of minors and referred to Weskoppies Hospital in terms of the Criminal Procedure Act over a period of 21 years. S Afr J Psychiatry. 2016;22(1):a887. https ://doi.org/10.4102/sajpsychiatry.v22i1.887.

110. Matete FG. Crime and mental illness in Africa: a retrospective study of court referrals to a provincial psychiatric clinic in Kenya. In: Okpaku SO, Okpaku SO, editors. Mental health in Africa and the Americas today: a book of conference proceedings. Nashville: Chrisolith Books; 1991. p. 223-9.

111. Mbassa DM. Characteristics of murder and attempted murder in Cameroon. Study based on expert psychiatric court reports. Med Trop. 2009;69(3):255-62.

112. Menezes SB. Parricides by mentally disordered offenders in Zimbabwe. Med Sci Law. 2010;50(3):126-30.

113. Menezes SB, Oyebode F, Haque MS. Mentally disordered offenders in Zimbabwe and in England and Wales: a socio-demographic study. Med Sci Law. 2007;47(3):253-61.

114. Odejide AO. Some clinical aspects of criminology: a study of criminal psychiatric patients at the Lantoro Psychiatric Institution. Acta Psychiatr Scand. 1981;63(3):208-24.

115. Offen $L$. The female offender and psychiatric referral: the medicalisation of female deviance? Med Law. 1986;5(4):339-48.

116. Ogunlesi AD, Makanjuola JD, Adelekan ML. Offenders admitted to the Neuro-Psychiatric Hospital Aro, Abeokuta: a ten-year review. West Afr Med J. 1988;7:129-35.

117. Prinsloo J, Hesselink A. Behavioural characteristics of offenders with mental health disorders in a South African prison population. J Psychol Africa. 2014;24(5):445-8.

118. Touari M, Mesbah M, Dellatolas G, Bensmail B. Association between criminality and psychosis: a retrospective study of 3984 expert psychiatric evaluations. Rev Epidemiol Sante Publique. 1993;41(3):218-27.

119. Verster IVRP. Mental disorders in patients referred for psychiatric observation after committing homicide. J Jurdical Sci. 1999:24(1):58-66.

120. Yusuf AJ, Nuhu FT. The profile of mentally ill offenders in Katsina, Northern-Nigeria. Afr J Psychiatry. 2009;12(3):231-2.

121. Zabow T. Psychiatric evidence in extenuation: assessment and testimony in homicide defendants. Med Law. 1989;8(6):631-9.

122. Atilola O. Different points of a continuum? Cross sectional comparison of the current and pre-contact psychosocial problems among the different categories of adolescents in institutional care in Nigeria. BMC Public Health. 2012;12:554.

123. Atilola O, Ola B, Abiri G, Adewuya AO. Correlations between psychopathology and self-reported quality of life among adolescents in youth correctional facilities in Lagos, Nigeria: a short report. Crim Behav Ment Health. 2017;28(1):28-35. https://doi.org/10.1002/cbm.2042.

124. Atilola O, Ola B, Abiri G, Sahid-Adebambo M, Odukoya O, Adewuya A, Coker O, Folarin O, Fasawe A. Status of mental-health services for adolescents with psychiatric morbidity in youth correctional institutions in Lagos. J Child Adolesc Ment Health. 2017;29(1):63-83.

125. Adegunloye OA, Yusuf AD, Ajiboye PO, Issa BA, Buhari ON. Prevalence and correlates of disruptive behavior disorders in Youths in a Juvenile Borstal Institution. Niger J Psychiatry. 2010;8:12-7.

126. Ajiboye PO, Yussuf AD, Issa BA, Adegunloye OA, Buhari ON. Current and lifetime prevalence of mental disorders in juvenile borstal institution in Nigeria. Res J Med Sci. 2009;3(1):26-30.

127. Issa BA, Yussuf AD, Ajiboye PO, Buhari OI. Prevalence of psychiatric morbidity among inmates of a borstal institution in Nigeria. Int J Prison Health. 2009:5(2):88-94. 
128. Yusuf ADAP, Issa BA, Buhari Ol. Prevalence and risk factors for psychiatric disorders among youths in a Borstal Institution in Nigeria. J Pak Psych Soc. 2011;8:22.

129. Olashore AA, Ogunwale A, Adebowale TO. Correlates of conduct disorder among inmates of a Nigerian Borstal Institution. Child Adolesc Psychiatry Ment Health. 2016;10:13.

130. Olashore AA, Akanni OO, Olashore OO. Associate factors of delinquency among incarcerated male juveniles in a borstal institution in Nigeria. Int J Forensic Ment Health. 2017:16(3):207-14.

131. Idemudia SE. The result of API and CCEI on Nigerian prisoners and relationship with the duration of confinement. IFE Psychol Int J. 1998;6(1):91-102.

132. Idemudia ES. Personality and criminal outcomes of homeless youth in a Nigerian jail population: results of PDS and MAACL-H assessments. J Child Adolesc Ment Health. 2007;19(2):137-45.

133. Ineme ME, Osinowo HO. Prior substance use, depression and gender as determinants of self-harm urges in prison inmates: a study of Uyo prison. Afr J Drug Alcohol Stud. 2016;15(1):61-73.

134. Odejide AO. Criminal justice and psychiatry: a study of the pattern of criminality in Ibadan division. Afr J Med Med Sci. 1979:8(1-2):41-4.
135. Sukeri K, Betancourt OA, Emsley R, Nagdee M, Erlacher H. Forensic mental health services: current service provision and planning for a prison mental health service in the Eastern Cape. Afr J Psychiatry. 2016;22(1):787.

136. Liddicoat R, Coulter MA, Fairbairn V. A personality adjustment questionnaire for the classification of psychopathic criminal offenders. Psychol Afr. 1972;14(3):200-15.

137. Prinsloo J, Ladikos A. Exploring the application of actuarial criminology in southern Africa. J Psychol Africa. 2007:17(1-2):67-72

138. Prinsloo J. Offenders with mental disorders in a South African prison population: profiling the behavioural characteristics on mental illness. J Psychol Africa. 2013;23(1):133-8.

139. Bunnting BG, Wessels WH, Lasich AJ, Pillay B. The distinction of malingering and mental illness in black forensic cases. Med Law. 1996;15(2):241-7.
Ready to submit your research? Choose BMC and benefit from:

- fast, convenient online submission

- thorough peer review by experienced researchers in your field

- rapid publication on acceptance

- support for research data, including large and complex data types

- gold Open Access which fosters wider collaboration and increased citations

- maximum visibility for your research: over $100 \mathrm{M}$ website views per year

At BMC, research is always in progress.

Learn more biomedcentral.com/submissions 